ARTICLE

https://doi.org/10.1038/s41467-019-12913-z

\title{
Early dorsomedial tissue interactions regulate gyrification of distal neocortex
}

Victor V. Chizhikov, Igor Y. Iskusnykh (10 ${ }^{1}$, Ekaterina Y. Steshina ${ }^{1}$, Nikolai Fattakhov (D) ${ }^{1}$, Anne G. Lindgren², Ashwin S. Shetty ${ }^{3}$, Achira Roy (10) ${ }^{4}$, Shubha Tole $^{3} \&$ Kathleen J. Millen ${ }^{4,5 \star}$

The extent of neocortical gyrification is an important determinant of a species' cognitive abilities, yet the mechanisms regulating cortical gyrification are poorly understood. We uncover long-range regulation of this process originating at the telencephalic dorsal midline, where levels of secreted Bmps are maintained by factors in both the neuroepithelium and the overlying mesenchyme. In the mouse, the combined loss of transcription factors Lmxla and $L m \times 1 b$, selectively expressed in the midline neuroepithelium and the mesenchyme respectively, causes dorsal midline Bmp signaling to drop at early neural tube stages. This alters the spatial and temporal Wnt signaling profile of the dorsal midline cortical hem, which in turn causes gyrification of the distal neocortex. Our study uncovers early mesenchymalneuroepithelial interactions that have long-range effects on neocortical gyrification and shows that lissencephaly in mice is actively maintained via redundant genetic regulation of dorsal midline development and signaling.

\footnotetext{
${ }^{1}$ Department of Anatomy and Neurobiology, University of Tennessee Health Science Center, Memphis, TN 38163, USA. ${ }^{2}$ Department of Human Genetics, University of Chicago, Chicago, IL 60637, USA. ${ }^{3}$ Department of Biological Sciences, Tata Institute of Fundamental Research, Mumbai, India. ${ }^{4}$ Center for Integrative Brain Research, Seattle Children's Research Institute, Seattle, WA 98101, USA. ${ }^{5}$ Department of Pediatrics, University of Washington, Seattle, WA 98101, USA. *email: vchizhik@uthsc.edu; kathleen.millen@seattlechildrens.org
} 
$\mathrm{N}$ eocortical gyrification, defined as folding of the outer pial surface and underlying neuronal layers of the brain but a smooth ventricular surface, results in an uneven regional thickness of the neocortex and is associated with enhanced species' cognitive abilities $^{1-4}$. Neocortical gyrification is a variable trait across mammalian species ranging from complete absence of gyrification in lissencephalic mammals, such as the mouse, to highly elaborate gyrification in many primates, such as humans. It is believed that the most recent common mammalian ancestor had a gyrencephalic (folded) cortex and that several transitions between gyrencephaly and lissencephaly (smooth cortex) happened during mammalian evolution as adaptations to each species' environment and lifestyle ${ }^{5-7}$.

Recent studies have revealed a coordinated sequence of neurogenic events in the developing neocortex of naturally gyrencephalic mammals, which begins with an early accumulation of apical cortical progenitors (APs) in the neocortical ventricular zone (VZ), followed by expansion of basal progenitors (BPs), including intermediate progenitors (IPs) and basal radial glia (bRG). BPs arise from APs and expand in the emerging gyri. It is believed that extensive generation of BPs, especially bRG, at the peak of neurogenesis and the subsequent production of neurons from these progenitors is a major force driving cortical gyrification in higher mammals ${ }^{3,8-12}$. A number of genes that promote gyrification acting locally in cortical progenitors have been identified $^{4,13-21}$. During development, however, many cortical features are regulated by both locally acting mechanisms and long-range signaling. The contribution of signaling molecules, in particular long-range signaling molecules, to cortical gyrification remains poorly understood. Shh and Fgf signaling are known to promote cortical gyrification ${ }^{16,22-24}$. In humans, Shh is expressed and acts locally in cortical progenitors, while the sources of gyrification-related Fgf signaling remain unknown ${ }^{16,22}$.

Although there have been extensive efforts devoted to identify the mechanisms that promote cortical folding in gyrencephalic mammals, the endogenous mechanisms that maintain lissencephaly (suppress gyrification) in naturally lissencephalic mammals have received little attention and remain virtually unknown. One possibility is that lissencephalic species simply lack genes or regulatory elements necessary to drive cortical gyrification ${ }^{13-15,18,19}$. Alternatively, genes in the genome of lissencephalic species actively maintain lissencephaly, suppressing gyrification. To date, several mouse models with a gyrencephalic neocortex have been described. The vast majority of these, however, have been generated via local or transient manipulation of gene function and, therefore, alone are insufficient to dissect the endogenous mechanisms that maintain lissencephaly in the mouse brain. The only exception is the Flrt1-/-;Flrt3-/- mouse model ${ }^{25}$. Dual loss of the adhesion genes Flrt1 and Flrt3 affects migration of differentiating neurons and results in folding of the mouse neocortex. In this mouse model, however, cortical folding develops without the predominant expansion of BPs observed in higher mammals ${ }^{25}$.

While studying the role of transcription factors Lmxla and Lmx1b in the cerebellum ${ }^{26}$, we observed that $L m \times 1 a / b$ double, but not single, mutants had unexpected neocortical gyrification. We found that neither $L m x 1 a$ nor $L m x 1 b$ were expressed in the neocortex. In contrast to more posterior central nervous system ${ }^{26}$, in the telencephalon, these two genes were not even coexpressed: Lmxla was expressed in the telencephalic dorsal midline neuroepithelium (DMe), while $L m x 1 b$ was expressed in head mesenchyme. Cortical gyrification in $\operatorname{Lm} \times 1 a-/-; b-/-$ mutants was associated with expansion of neocortical BPs and resulted from the disruption of spatial and temporal dynamics of Bmp and Wnt signaling cascades originating at the distantly located dorsomedial telencephalon. Our study identifies an unexpected role of dorsal midline signaling in the long-range regulation of cortical gyrification and shows that mesenchymal-neuroepithelial interactions are necessary to maintain lissencephaly in mice.

\section{Results}

Cortical gyrification in $\operatorname{Lm} x \mathbf{l a}-/-; b-/-$ mice. Transcription factors Lmxla and Lmxlb redundantly regulate development of several cellular populations during embryonic development, such as the hindbrain roof plate and midbrain dopaminergic neurons ${ }^{26,27}$. While studying the role of $\operatorname{Lm} x 1$ genes in cerebellar development ${ }^{26}$, we found that $\operatorname{Lm} \times 1 a-/-; b-/-$ mutants had unexpected and striking gyrification of the neocortex (Fig. 1a-f, Supplementary Fig. 1a-d). All 12 Lmx $1 a-/-; b-/-$ mutants that we dissected at late embryonic stages had macroscopic cortical folds apparent in both left and right hemispheres (Fig. 1b) yet the cortex remained lissencephalic in littermates with loss of either single gene $(n=20-25$ mutants for each genotype) (Supplementary Fig. 2). Analysis of postnatal stages was precluded by neonatal death caused by a requirement for $L m \times 1 b$ during mid/ hindbrain development ${ }^{26}$. To compare location of cortical gyri in different e18.5 embryos, we divided the neocortical ventricular surface into 10 equally sized segments and measured cortical thickness in the middle of each of these segments (Supplementary Fig. 1e). Average cortical thickness was similar throughout wild-type cortex yet was uneven between different regions of Lmxla-/-;b-/- neocortex, illustrating non-random locations of cortical gyri (Supplementary Fig. 1g, h). In both hemispheres, the most prominent was a dorsal gyrus located in cortical segments 2 (and also in segment 3 in right hemisphere), while the neuroepithelium in segment 4 (and also in segments 5 and 6 in right hemisphere) was thinner. More ventral gyri were less prominent (Supplementary Fig. 1b-d, h). Consistent with cortical gyrification, neocortical area (measured between the dorsal cortical bend and lateral ganglionic eminence, LGE) was increased in Lmx1a-/-;b-/- mutants compared to wild-type littermates (Supplementary Fig. 1f).

Similar to wild-type littermates ${ }^{28}$, in $L m \times 1 a-/-; b-/-$ mutants, Cux $1+$ (upper layer) neurons occupied more superficial positions relative to Ctip2+ (lower layer) neurons, revealing largely preserved cortical lamination (Fig. 1c, d). Thus, Lmxla and $L m x 1 b$ function redundantly to maintain lissencephaly in the mouse, and simultaneous loss of these genes is sufficient to induce gyrification of the mouse cortex.

Aberrant expansion of progenitors in $\operatorname{Lmx} 1 a-/-; b-/-$ cortex. To determine whether cortical gyrification in $L m \times 1 a-/-; b-/-$ mutants was associated with expansion of cortical BPs, we analyzed embryos at e15.5, during mid-neurogenesis, when Lmx1a-/-;b-/- double mutant cortex first displays evidence of gyrification (cortical plate folding relative to ventricular surface) (Fig. 1e, f). In the emerging cortical gyri, but not sulci, of e15.5 Lmx1a-/-;b-/- mutants, more Pax6+/Tbr2 - and Tbr2+ cells were observed in basal locations relative to wild-type controls (Fig. 1g-n), consistent with focal expansion of bRG and IPs $16,29,30$.

Since proliferation of BPs and the subsequent production of neurons from these progenitors are major mechanisms of cortical gyrification in higher mammals ${ }^{3,8-12}$, we studied BPs in the emerging cortical gyri of $\operatorname{Lmx} 1 a-/-; b-/-$ mutants in more detail. Co-labeling with a progenitor marker Ki67 confirmed that basal Pax6+/Tbr2 - and Tbr2+ cells in Lmx1a-/-;b-/mutants were indeed progenitors (Supplementary Fig. 3a, b). A short $(90 \mathrm{~min})$ BrdU pulse was used to label proliferating cells in the S-phase of the cell cycle. This analysis revealed numerous basally located BrdU+ cells in the emerging gyri of 

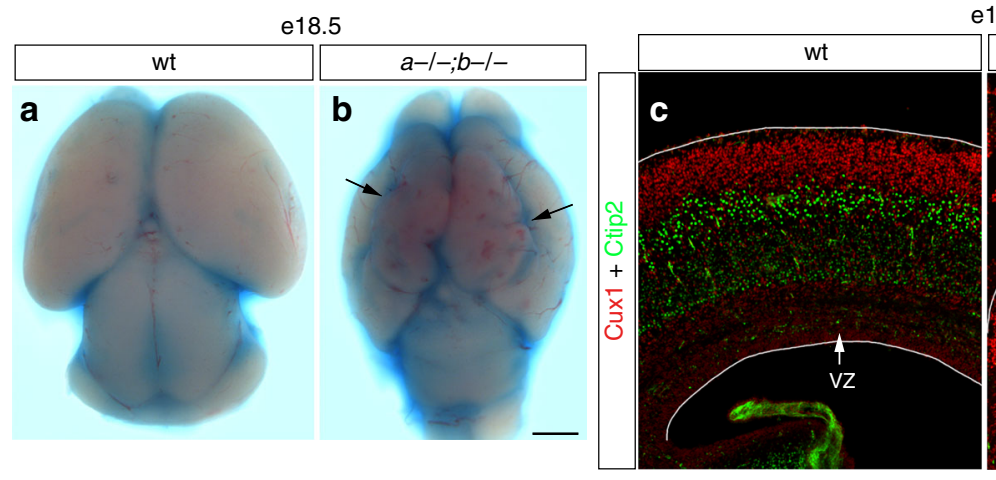

e18.5
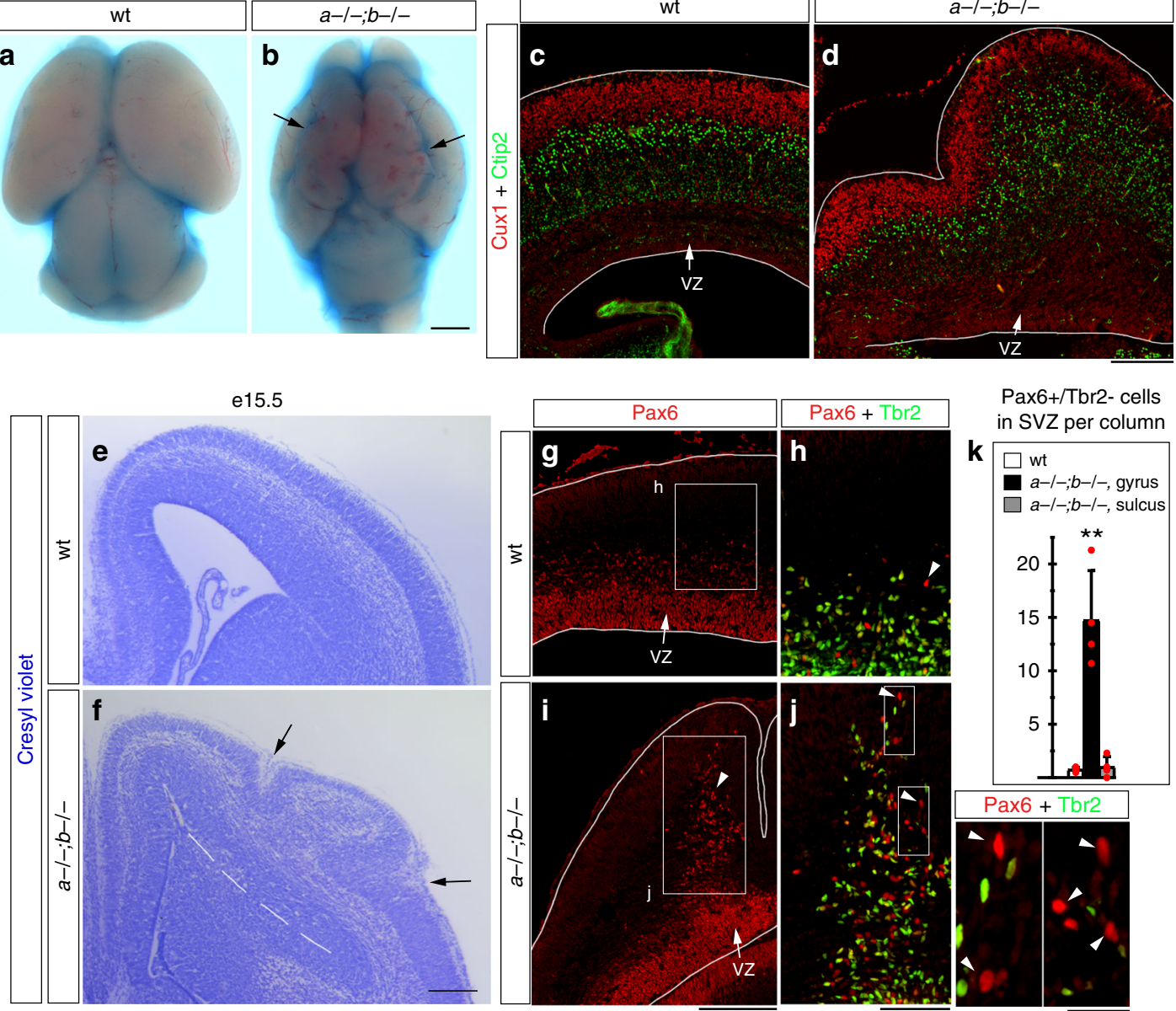

Pax6+/Tbr2- cells in SVZ per column
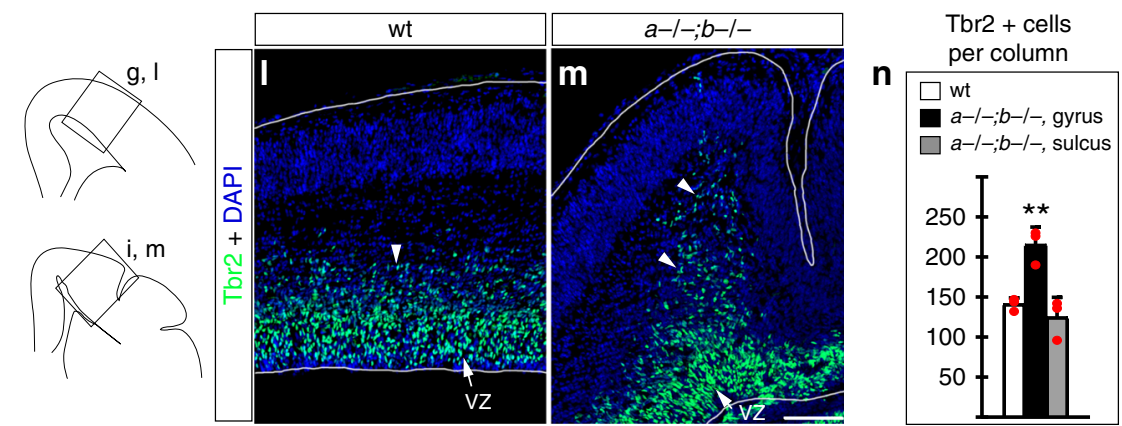

Fig. 1 Gyrification with BPs expansion in the $L m \times 1 a-/-; b-/-$ mouse cortex. Dorsal view of whole mount telencephalon with fast green dye applied on the telencephalic surface $(\mathbf{a}, \mathbf{b})$ and coronal cresyl violet or antibody-stained sections $(\mathbf{c}-\mathbf{j}, \mathbf{I}, \mathbf{m})$ at indicated stages. $\mathbf{a}, \mathbf{b}$ Cortical surface of e18.5 Lmx1a-/-;b-/ - mutants, but not wild-type littermates, showed groves (arrows) that extended along the anterior-posterior axis in each cortical hemisphere. c, d Cux1/Ctip2-immunostained sections showing that despite gyrification of the outer cortical surface (faces up), cortical layering was not grossly disrupted in $L m \times 1 a-/-; b-/-$ mutants. Corresponding ventricular surface (faces down) was not folded. VZ - ventricular zone. e, f Arrows point to sulci that develop on the outer cortical surface of $L m \times 1 a-/-; b-/-$ embryos at e15.5 (f). Dashed line demarcates cortical ventricular surface and lateral ganglionic eminence. g-k Numerous basally located Pax6+ cells were present in the emerging gyri (i, arrowhead) but not sulci in e15.5 Lmx1a-/-;b-/mutants (i). The number of Pax6+/Tbr2 - cells located basal to the dense band of Tbr2 + IPs (arrowheads in $\mathbf{h}, \mathbf{j}$, j insets) was dramatically increased in cortical gyri but not sulci relative to wild-type littermates ( ${ }^{\star \star} p<0.01, n=4$ embryos per genotype). I-n In e15.5 Lmx $1 a-/-; b-/-$ mutants, Tbr2 + IPs (arrowheads) were increased in the number in cortical gyri but not sulci relative to wild-type littermates ( ${ }^{\star \star} p<0.01, n=3$ embryos per genotype). All data are mean \pm s.d., all $p$ values are from two-tailed $t$-test after Bonferroni correction for multiple comparisons. Source data for panels $\mathbf{k}$ and $\mathbf{n}$ are provided as Source Data File. Scale bars: $1 \mathrm{~mm}(\mathbf{a}, \mathbf{b}) ; 200 \mu \mathrm{m}(\mathbf{c}-\mathbf{g}, \mathbf{i}) ; 100 \mu \mathrm{m}(\mathbf{h}, \mathbf{j}, \mathbf{I}, \mathbf{m}) ; 25 \mu \mathrm{m}$ (insets in panel $\mathbf{j}$ )

Lmx1a-/-;b-/- embryos (Supplementary Fig. 3c, g, arrowheads). The fraction of basally located BrdU+ cells was significantly higher in the emerging gyri of $L m x 1 a / b$ double mutants compared to wild-type controls (Supplementary Fig. 3c, g, k) arguing that similar to naturally gyrencephalic mammals ${ }^{3,9,11}$, in $L m x 1 a-/-; b-/-$ cortical gyri, proliferation was shifted to basal positions. Triple BrdU/Pax6/Tbr2 labeling revealed that in $L m \times 1 a-/-; b-/-$ mutants, many BPs incorporated BrdU and, thus, were proliferating (Supplementary Fig. $3 c-e, g-i)$. There was a significant increase in the numbers 
of both S-phase BrdU+/Pax6+/Tbr2- bRG and BrdU+/Tbr2+ IPs, but not apical BrdU+/Pax6+ cells in cortical gyri of Lmx1a-/-;b-/- mutants (Supplementary Fig. 3c-f, g-j, l-n). Since Pax6+/Tbr2 - bRG cells were very rare in wild-type embryos, we were unable to compare the fraction of BrdU+ (Sphase) bRG cells between wild-type and Lmx1a-/-;b-/embryos. However, we found that a similar fraction ( 20\%) of Tbr2+ IPs were BrdU+ (S-phase) in both wild-type and Lmx1a-/-;b-/- cortex (Supplementary Fig. 3o), suggesting a similar proliferation rate ${ }^{31}$ of IPs in wild-type and $L m x 1 a-/-$; $b-/-$ mutants.

A longer $(20 \mathrm{~h})$ BrdU pulse revealed an increased fraction of progenitors exiting the cell cycle in nascent gyri of $L m \times 1 a-/-$; $b-/-$ embryos (Supplementary Fig. 3p-r), consistent with increased neurogenesis ${ }^{23}$. Analysis of $\operatorname{Lmx} 1 a-/-; b-/-$ mutants at e15.5, the time of gyri development, revealed a similar density of apoptotic (activated Caspase 3+) cells in cortical areas producing gyri and sulci (Supplementary Fig. 4), indicating that localized increase in apoptosis is not a major mechanism of cortical gyrification in $\operatorname{Lm} x 1 a-/-; / b-/-$ mutants. Thus, cortical gyrification in $L m \times 1 a-/-; b-/-$ mutants was associated with expansion of cortical BPs and increased neurogenesis.

Next, we analyzed e12.5 embryos, at the beginning of cortical neurogenesis when $L m \times 1 a-/-; b-/-$ mutants were not yet gyrified (Fig. 2a, b). We studied progenitor populations in two cortical regions-cortical segment 2 (the most consistent location of a future gyrus) and cortical segment 4 (a region with the lowest cortical thickness at e18.5) (Fig. 2c, Supplementary Fig. 1h). At this early stage, compared to controls, both cortical regions of the double mutants had increased numbers of Pax6+ APs, increased radial thickness of the VZ (Fig. $2 \mathrm{~d}-\mathrm{i}$ ), reduced numbers of Tbr2+ IPs (Fig. 2j-o), and reduced cell-cycle exit (Fig. 2p-u). Thus, in Lmx $1 a / b$ double mutants, global overproduction of APs precedes local accumulation of BPs at later stages.

$L m x 1 a / b$ are not coexpressed in the developing telencephalon. We expected the LmxIa and $L m x 1 b$ to be coexpressed in the developing telencephalon, based on their co-expression in other cellular populations redundantly regulated by these genes ${ }^{26,27}$. Our extensive in situ hybridization, immunohistochemical and fate-mapping analyses revealed, however, that these genes are never coexpressed in the developing telencephalon. Further, neither is even expressed in neocortical neuroepithelium. $L m \times 1 a$ expression was always restricted to the telencephalic DMe, beginning at the tips of the neural folds at e8.5 and through differentiation of the DMe into the medial choroid plexus epithelium $(\mathrm{ChPe})$ and the cortical hem $(\mathrm{CH}) . L m x 1 b$ expression was restricted to the head mesenchyme, including the mesenchyme located adjacent to the DMe (Fig. 3a, b, Supplementary Figs. 5 and 6). Loss of one $\operatorname{Lmx} 1$ gene did not alter expression pattern of the remaining $L m x 1$ gene (Supplementary Fig. 7). When considered together with the Lmx1a-/-;b-/- cortical phenotype, the $L m x I a$ and $L m x 1 b$ expression pattern suggests that redundant $L m x 1 a / b$-dependent mesenchymal and $\mathrm{DMe}$ signaling pathways regulate gyrification of distal neocortex.

Aberrant DMe and Wnt signaling in Lmx1a-/-;b-/mutants. By e12.5, the wild-type telencephalic DMe differentiates into the medial $\mathrm{Ttr}+\mathrm{ChPe}$ and adjacent $\mathrm{CH}$ that expresses secreted Wnt3a $\mathrm{a}^{32-34}$ (Fig. 3c, e). In Lmx1a-/-;b-/- mutants at this stage, however, Ttr expression was absent in a thickened DMe while Wnt $3 a$ expression expanded across the presumptive ChPe, resulting in an enlarged Wnt $3 a$ expression domain at the dorsal midline compared to controls (Fig. 3c-f). This phenotype was not observed in either single-gene mutant (Supplementary
Fig. 8). In double mutants, the thickened medial neuroepithelium showed increased proliferation in the presumptive ChPe as early as at e10.5 (Supplementary Fig. 9a-g). By e15.5, there was partial amelioration of Lmxla-/-;b-/- DMe deficits: Ttr ChPe expression was present and $W n t 3 a$ expression was restricted to the $\mathrm{CH}$ (Fig. $3 \mathrm{~g}-\mathrm{j}$ ), although $\mathrm{ChPe}$ proliferation was still elevated compared to controls (Supplementary Fig. 9h, i). The double mutant $\mathrm{CH}$ also displayed increased apoptosis (Supplementary Fig. 9j-1), resulting in a small $W n t 3 a+\mathrm{CH}$ at e15.5 (Fig. 3g, h).

Early expansion of $W n t 3 a+\mathrm{CH}$ in e12.5 Lmxla-/-;b-/mutants was associated with elevated nuclear $\beta$-catenin, a definitive readout of canonical Wnt signaling ${ }^{35}$ (Supplementary Fig. 10) and elevated expression of downstream Wnt- $\beta$-catenin target genes Lef1 and Axin $2^{36}$ in the neocortical primordium (Supplementary Fig. 11). However, at e15.5, consistent with the dramatic reduction of size of the double-mutant $W n t 3 a+\mathrm{CH}$, nuclear $\beta$-catenin was undetectable in the $L m \times 1 a-/-; b-/-$ neocortex, and Lef1 and Axin2 levels were significantly reduced relative to comparably staged controls (Supplementary Fig. 12). These data define two distinct phases of canonical Wnt signaling and reception in the $\operatorname{Lm} x 1 a-/-; b-/-$ embryonic telencephalon: an increased early phase and a decreased late phase.

In addition to producing Wnt signaling molecules, the early $\mathrm{DMe}$ (both the $\mathrm{CH}$ and $\mathrm{ChPe}$ ) gives rise to Cajal-Retzius (CR) cells, a transient neuronal population that migrates along the outer surface of cortical neuroepithelium and regulates cortical development ${ }^{37,38}$. In $\operatorname{Lm} \times 1 a-/-; b-/-$ mutants, similar to wildtype embryos, CR cells uniformly covered the neocortical primordium, suggesting that cortical gyrification in these mutants does not directly arise from CR cells migratory defects (Supplementary Fig. 13). Thus, to define the mechanism of Lmxla-/-;b-/- cortical gyrification, we focused on misregulated DMe-derived Wnt signaling.

Lmx1a-/-;b-/- cortical gyrification depends on Wnt signals. Wnt/ $\beta$-catenin signaling promotes expansion of cortical APs and suppresses the generation of BPs from $\mathrm{APs}^{29,31,39}$. Early constitutive ablation of $\beta$-catenin in the developing telencephalon increased the numbers of both bRG and IPs ${ }^{29}$, the phenotype we also observed in $L m \times 1 a-/-; b-/-$ mutants. However, in contrast to $L m \times 1 a-/-; b-/-$ embryos, $\beta$-catenin-ablated mice did not show cortical gyrification, and IP expansion in these mice was observed only at the beginning (e12.5) but not in the middle (e14.5) of neurogenesis, likely due to precocious depletion of the AP pool ${ }^{29}$.

A key difference between $\beta$-catenin-ablated mice and our Lmxla-/-;b-/- mutant embryos is that in contrast to $\beta$ catenin-ablated mice, in $L m \times 1 a-/-; b-/-$ mutants, a dynamic decrease in Wnt- $\beta$-catenin signaling followed its transient overactivation and the early overexpansion of APs. To balance early high levels of Wnt signaling in Lmxla-/-;b-/- neocortex, we electroporated a plasmid encoding Dkk1, a secreted Wnt inhibitor ${ }^{39}$ into the e12 $L m \times 1 a-/-; b-/-$ neocortical primordium. This was sufficient to rescue the gyrification phenotype and restore a lissencephalic cortex on the electroporated side, when assessed at e18 (Fig. 4a). Dkk1-electroporated Lmx1a-/-;b-/hemispheres had significantly lower numbers of APs, bRG, and IPs compared to $\operatorname{Lm} \times 1 a-/-; b-/-$ cortices electroporated with GFP alone (Fig. 4b-h). Thus, we conclude that aberrant dynamics of Wnt signaling is a major contributor to neocortical gyrification in $L m \times 1 a-1-; b-/-$ mice.

$L m x 1 a / b$ regulate the DMe Wnt source via $\mathrm{Bmp}$ signaling. Next, we focused our attention on the early development of the DMe Wnt source. We hypothesized that $L m x 1 b$ regulates 


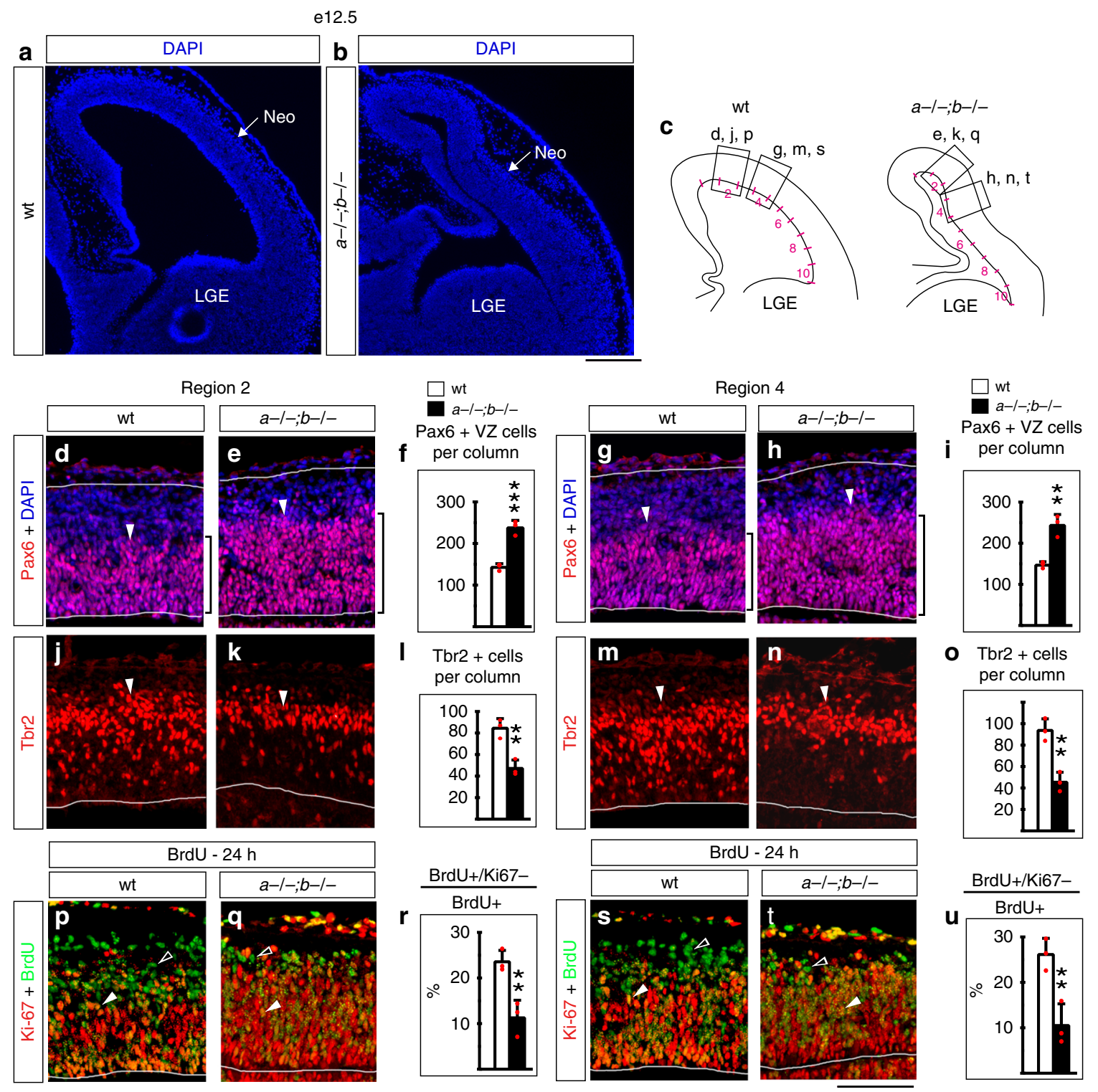

Fig. 2 Accumulation of APs precedes gyrification of the $L m \times 1 a-/-; b-/-$ cortex. Coronal sections at e12.5. a, b No evidence of cortical gyrification (outgrowths on outer cortical surface without bending of the corresponding ventricular surface) was detected in $L m \times 1 a-/-; b-/-$ mutants at e12.5. Neoneocortical primordium. LGE lateral ganglionic eminence. c Ventricular surface in wild-type and $L m \times 1 a-/-; b-/-$ neocortical primordium (between the dorsal cortical bend and LGE) was subdivided into 10 equally sized regions (segments 1-10) and cells were quantified in segment 2 (d-f $\mathbf{f} \mathbf{j}-\mathbf{I}, \mathbf{p}-\mathbf{r}$ ) and segment 4 (g-i, $\mathbf{m}-\mathbf{o}, \mathbf{s}-\mathbf{u}) . \mathbf{d}-\mathbf{i}$ Pax6+ APs (d, e, $\mathbf{g}$, h, arrowheads) were increased in the number $(\mathbf{f}, \mathbf{i})\left({ }^{\star \star *} p<0.001\right.$ and $\left.{ }^{\star *} p<0.01\right)$ and Pax6+VZ zone (bracket) was thicker in $L m \times 1 a-/-b-/-$ mutants in both segments of the neocortical primordium. $\mathbf{j}-\mathbf{o}$ Tbr2+ IPs (j, $\mathbf{k}, \mathbf{m}, \mathbf{n}$, arrowheads) were reduced in the number $(\mathbf{I}, \mathbf{0})\left({ }^{\star \star} p<0.01\right)$ in $L m \times 1 a-/-; b-/-$ mutants in both segments of the neocortical primordium. p-u Mice were injected with BrdU $24 \mathrm{~h}$ prior to collecting embryos. p, q, s, t Arrowheads point to cells that re-entered the cell cycle (BrdU+/Ki67+ cells), open arrowheads-to cell that exited the cell cycle (BrdU + /Ki67- cells). A lower fraction of cells exited the cell cycle (the number of BrdU+/Ki67 - cells divided by the number of BrdU+ cells) ( ${ }^{\star \star} p<$ $0.01)$ in $L m \times 1 a-/-; b-/-$ mutants in both segments of the neocortical primordium. All data are mean \pm s.d., all $p$ values are from two-tailed $t$-test, $n=3$ embryos per genotype. Source data for panels $\mathbf{f}, \mathbf{i}, \mathbf{I}, \mathbf{0}, \mathbf{r}, \mathbf{u}$ are provided as Source Data File. Scale bars: $200 \mu \mathrm{m}(\mathbf{a}, \mathbf{b}) ; 100 \mu \mathrm{m}$ (all other panels)

expression of secreted factors that diffuse from the medial head mesenchyme and cooperate with a Lmxla-dependent pathway in the DMe to regulate the temporal and spatial patterning of the $\mathrm{ChPe} / \mathrm{CH}$. Using a candidate gene approach, in situ hybridization revealed $B m p 4$ to be expressed in both the $L m x 1 b$ (mesenchymal) and $L m x 1 a$ (DMe) territory in wild-type embryos (Fig. 5a, b). Loss of $L m \times 1 b$ reduced Bmp4 expression in the medial mesenchyme but did not alter expression of this gene in the DMe or lateral mesenchyme (Fig. 5b, d, e; Supplementary Fig. 14). Based on in situ hybridization, loss of Lmxla did not compromise Bmp4 expression in either compartment (Fig. 5b, c, e; Supplementary Fig. 14).
Although in situ hybridization is well suited for spatial distribution of gene expression, it is not strictly quantitative. We, therefore, used QRT-PCR analysis of laser capture microdissected dorsal mesenchyme and DMe to more quantitatively assess expression of Bmp genes in each compartment (Fig. 5a, f; Supplementary Fig. 15). This analysis revealed that loss of Lmxla decreased expression levels of both $B m p 4$ and related Bmp2 in the DMe. It also confirmed that loss of $L m x 1 b$ alone caused drastic decrease of Bmp4 as well as Bmp2 expression in medial mesenchyme. In double mutants, Bmp4/2 mesenchymal expression levels were similar to those in $L m x 1 b$ mutants, while double-mutant $\mathrm{DMe}$ 

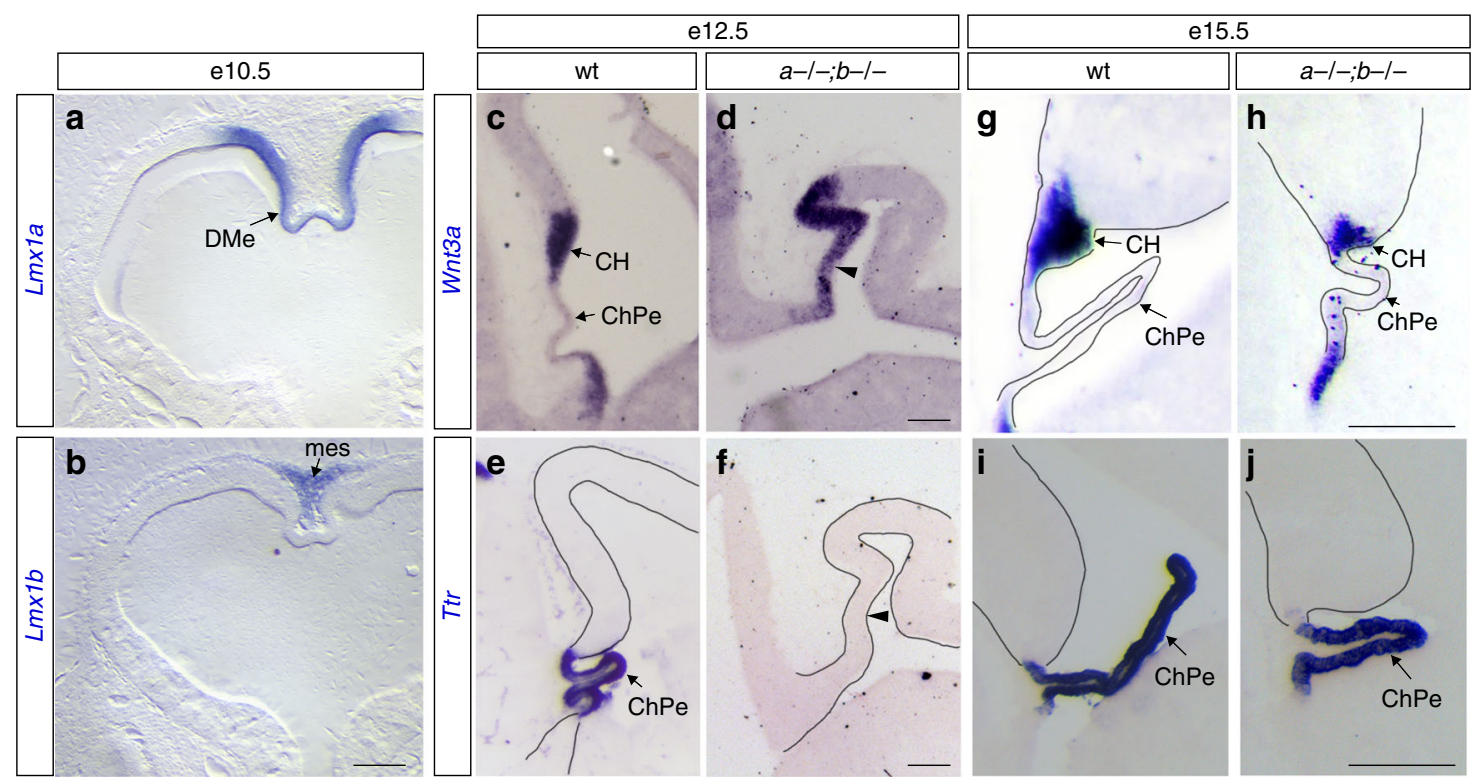

Fig. 3 Dual loss of complementary expressed $L m \times 1 a / b$ causes transient DMe mispatterning. $\mathbf{a}$, $\mathbf{b}$ In situ stained wild-type coronal telencephalic sections at e10.5 showing specific expression of $L m \times 1 a$ in the DMe and $L m x 1 b$-in medial mesenchyme (mes.). c-f At e12.5, wild-type $\mathrm{CH}$ expressed Wnt3a (c), while $\mathrm{ChPe}-\operatorname{Ttr}(\mathbf{e})$. In Lmx1a-/-;b-/- mutants, Wnt3a expression domain was enlarged, expanding into the prospective ChPe territory (d, arrowhead), while Ttr was not expressed (f, arrowhead). g-j DMe patterning was restored in e15.5 Lmx1a-/-;b-/- mutants, with Ttr expression in the ChPe and Wnt3a-in the $\mathrm{CH}$. Wnt3a+ CH domain, however, was abnormally small $(\mathbf{g}, \mathbf{h})$. Scale bars: $200 \mu \mathrm{m}(\mathbf{a}, \mathbf{b}, \mathbf{g}, \mathbf{h}) ; 150 \mu \mathrm{m}(\mathbf{c}-\mathbf{f}) ; 250 \mu \mathrm{m}(\mathbf{i}, \mathbf{j})$

Bmp4/2 expression levels were similar to those in $L m x 1 a$ mutants (Fig. 5f; Supplementary Fig. 15). A definitive readout of Bmp signal reception, pSmad immunostaining intensity ${ }^{33}$, was significantly decreased in the DMe of double but not single-gene mutants at e10.5 (Fig. 5g-k).

We hypothesized that dual loss of $L m \times 1 a$ and $L m \times 1 b$ reduced early Bmp signaling levels below a critical threshold required to pattern and maintain normal DMe and hence subsequent lateral cortical development. We, therefore, selectively electroporated Bmp4 into the DMe territory of $\operatorname{Lm} \times 1 a-/-; b-/-$ embryos at e10.5. At e12.5 the double mutant DMe was rescued as demonstrated by the reappearance of $\operatorname{Ttr}$ (Fig. 6a-d; Supplementary Fig. 16a, b) and reduction of abnormally high proliferation in the ChPe (Supplementary Fig. 17). Ectopic Wnt3a expression in the double-mutant ChPe was also lost and low levels of Wnt signaling were restored in the distal cortical primordium as revealed by analysis of expression of downstream Wnt- $\beta$-catenin target gene Axin2 (Supplementary Fig. 16). At e15.5, the cortical plate of electroporated $\operatorname{Lm} \times 1 a-/-; b-/-$ mutants was lissencephalic, with no evidence of gyrification (Fig. 6e, f). Thus, supplementing Bmp at the DMe was sufficient to normalize DMe development and Wnt signaling and suppress gyrification of the distantly located cortex in Lmx1a-/-;b-/- embryos. We conclude that $L m \times 1 a$ and $L m \times 1 b$ regulate DMe development via Bmp signaling and that misregulation of the DMe development and its Wnt signaling is central to the $\operatorname{Lm} \times 1 a-/-; b-/-$ cortical gyrification phenotype.

\section{Discussion}

Wnt signaling from medially located $\mathrm{CH}$ induces hippocampus and regulates cortical size, while mesenchymal signaling regulates proliferation of cortical APs and differentiation and migration of a subset of cortical neurons ${ }^{40-46}$. Here we show that simultaneous loss of two transcription factors, Lmxla, expressed in the telencephalic DMe, and Lmx1b, expressed in head mesenchyme, causes folding of the mouse cerebral cortex, implicating head mesenchyme and DMe in the regulation of cortical gyrification. Our data demonstrate that $L m x 1 a$ and $L m \times 1 b$ normally act to suppress cortical gyrification in the mouse via Bmp-dependent regulation of $\mathrm{DMe}$ development and its $\mathrm{Wnt}$ signaling dynamics (Fig. 7).

Loss of $L m x 1 a$ and $1 b$ reduced expression of genes encoding secreted Bmp4/2 molecules in the DMe and adjacent mesenchyme, respectively, decreasing overall levels of Bmp signaling in the DMe of Lmx1a-/-;b-/- mutants. It has been previously shown that complete loss of Bmp signaling in Bmp receptor $1 \mathrm{a}$ and $1 b$ double-mutant mice resulted in failure to induce both the $\mathrm{ChPe}$ and $\mathrm{CH}^{34}$. Reduced Bmp signaling in $L m \times 1 a / b$ double mutant, however, resulted in a qualitatively different phenotype. Specifically, we observed initial mispatterning of the DMe with delayed differentiation of the ChPe and expansion of Wnt3a expression across the prospective $\mathrm{ChPe}$ territory. Later during development, contraction of the $W n t 3 a+\mathrm{CH}$ domain occurred as apoptosis was elevated and a delayed $T t r+\mathrm{ChPe}$ developed in the Lmx1a-/-;b-/- DMe (Fig. 7). Exogenous medial Bmp4 rescued DMe patterning in $L m \times 1 a-/-; b-/-$ embryos, directly implicating downregulated Bmp signaling in the mutant phenotype. Thus, our studies demonstrate that DMe development is particularly sensitive to the level of Bmp signaling in vivo. Previous in vitro analysis revealed that high Bmp levels are needed to induce the ChPe fate ${ }^{47}$. While low Bmp signaling in $L m \times 1 a-/-$; $b-/-$ mutants was sufficient to induce $W n t 3 a+\mathrm{CH}$ by e12.5, delayed differentiation of $\mathrm{ChPe}$ until later developmental stages potentially reflects a delay in accumulation of threshold levels of Bmp signaling necessary for ChPe development. Regardless, our study revealed that Bmp signaling is required not only for the initial induction of the $\mathrm{CH}$ but also regulates its size and, thus, the temporal dynamics of DMe-derived Wnt3a signaling throughout development. Additionally, we identify that Bmp proteins produced both locally in the DMe and in the adjacent mesenchyme participate redundantly in DMe development.

Expansion of BPs and subsequent production of neurons from these progenitors has been identified as a major driving force of cortical gyrification in naturally gyrencephalic mammals. During development, BPs are generated from APs, and a typical neurogenic sequence in gyrencephalic mammals includes initial 

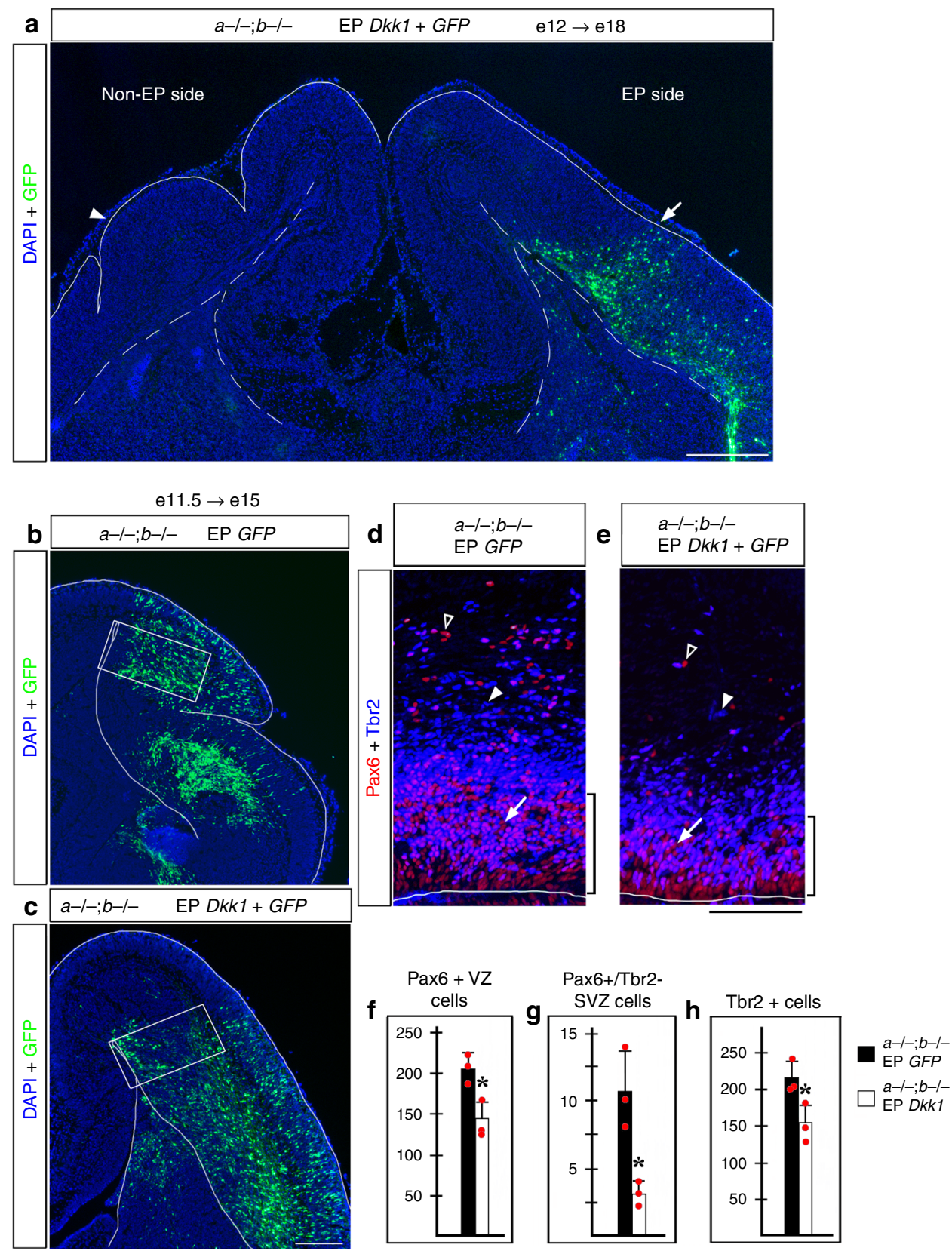

Fig. 4 Electroporation of $D k k 1$ suppresses Lmx1a-/-;b-/- cortical gyrification. a An Lmx1a-/-;b-/- embryo in utero electroporated with Dkk1+ GFP at e12 and analyzed at e18. Early electroporation of Dkk1 into neocortical primordium restored lissencephaly (arrow), while the non-electroporated side remained gyrencephalic (arrowhead). b-h Lmx1a-/-;b-/- mutants electroporated with Dkk1+ GFP or GFP alone at e11.5 and analyzed at e15. Panels $\mathbf{d}, \mathbf{e}$ are from sections adjacent to those shown in $\mathbf{b}, \mathbf{c}$ and correspond to regions boxed in $\mathbf{b}, \mathbf{c}$. $\mathbf{d}-\mathbf{h}$ In Dkk1-electroporated double mutants, the number of Pax6+ APs (arrows), Pax6+/Tbr2- bRGs (open arrowheads) and Tbr2+ IPs (arrowheads) was reduced compared to GFP-electroporated double mutants $\left({ }^{\star} p<0.05\right.$, two-tailed $t$-test, $n=3$ Dkk1-electroporated and $n=3$ GFP-electroporated $L m \times 1 a-/-; b-/-$ mutants). Bracket (d, e) indicates thickness of the Pax6+VZ, which was also reduced in Dkk7-electroporated $L m \times 1 a-/-; b-/-$ mutants. All data are mean \pm s.d. Source data for panels $\mathbf{f}-\mathbf{h}$ are provided as Source Data File. Scale bars: $400 \mu \mathrm{m}(\mathbf{a}) ; 200 \mu \mathrm{m}(\mathbf{b}, \mathbf{c}) ; 100 \mu \mathrm{m}(\mathbf{d}, \mathbf{e})$

expansion of APs followed by an extensive production of bRG and IPs during later peak of neurogenesis ${ }^{3,8-12}$. Expansion of APs in the VZ, although necessary for BPs production, does not automatically lead to excessive generation of BPs, which need to delaminate from the $\mathrm{VZ}^{4,11,48}$. In the cortical VZ, APs expand via symmetric divisions, while asymmetric divisions of APs result in the production of $\mathrm{BPs}^{11,29}$.

Wnt/ $\beta$-catenin signaling expands APs by promoting symmetric divisions and inhibiting asymmetric divisions, at least partially by controlling mitotic spindle orientation ${ }^{29}$. In contrast, reduction of Wnt signaling favors asymmetric cell divisions, activating the production of BPs (both bRG and IPs) from APs, depleting the AP pool ${ }^{29,39}$. Wnt/ $\beta$-catenin signaling, however, has not previously been considered a regulator of cortical gyrification because neither constitutive gain-of-function nor lossof-function $\mathrm{Wnt} / \beta$-catenin mouse models showed bona fide cortical gyrification $3,11,29,31,39$. Constitutive overexpression of a transgene encoding stabilized $\beta$-catenin in neural precursors produced a pseudo-gyrified cortex with both the ventricular and pial surfaces equally folded ${ }^{31}$, rather than the asymmetric 


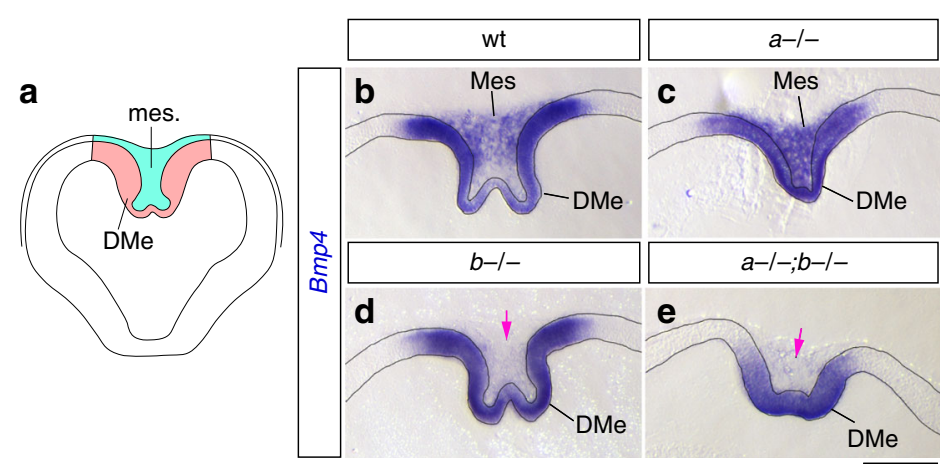

Bmp4 expression (LCM - QRT-PCR)
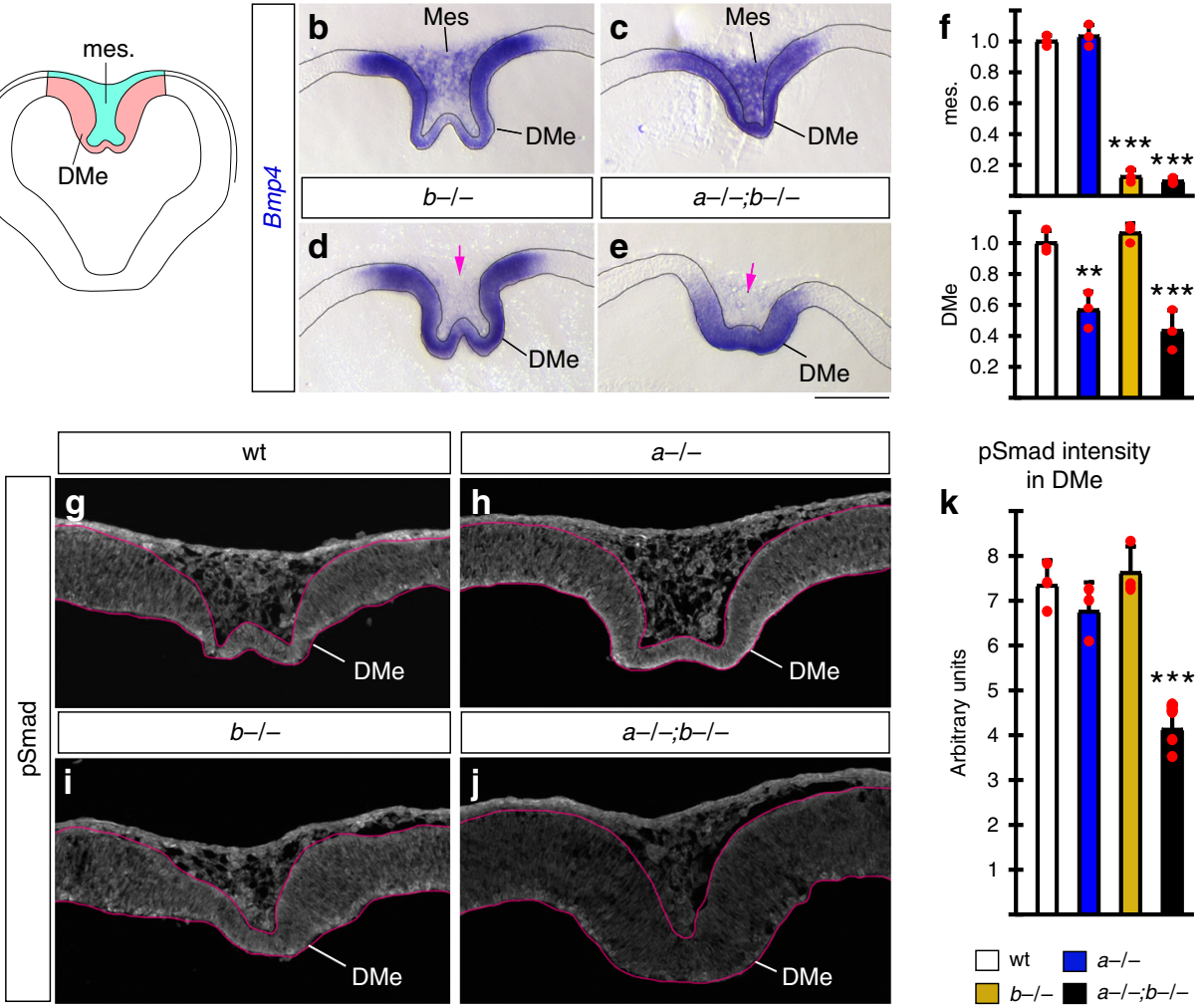

pSmad intensity

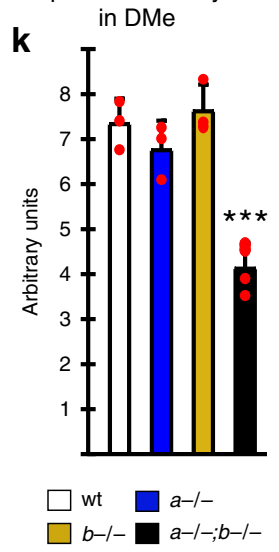

Fig. $5 \mathrm{Lmx} 1 a$ and $L m \times 1 b$ regulate Bmp signaling by controlling expression of Bmp4. a Schematic of e10.5 coronal telencephalic section showing DMe (red) and medial mesenchyme (green). b-e High magnification images of dorsal midline region of e10.5 telencephalic coronal sections stained with Bmp4 in situ probe. (Low magnification images of the same sections are shown in Supplementary Fig. 14.) b In wild-type telencephalon, Bmp4 was expressed in the DMe and medial mesenchyme adjacent to DMe (mes.). c-e Loss of $L m x 1 b$ abolished Bmp4 expression in medial mesenchyme (pink arrows in d, e) but not DMe. Based on in situ, loss of $L m x 1 a$ did not obviously reduce Bmp4 expression. $\mathbf{f}$ qRT-PCR analysis of DMe (red region in panel a) and medial mesenchyme (green region in panel a) isolated by laser capture microdissection (LCM). Based on qRT-PCR, loss of $L m x 1 b$ reduced expression of Bmp4 in medial mesenchyme (mes.) ( ${ }^{\star \star \star} p<0.001$ for $L m x 1 b-/-$ versus wild type and $L m \times 1 a-/-; b-/-$ versus wild-type comparisons, one-way ANOVA with Tukey's post hoc test, $n=3$ embryos per genotype). Loss of $L m x 1 a$ reduced expression of Bmp 4 in the DMe (** $p<0.01$ for $L m x 1 a-/-$ versus wild type and ${ }^{\star \star \star} p=$ 0.001 for $L m \times 1 a-/-; b-/-$ versus wild-type comparisons, one-way ANOVA with Tukey's post hoc test, $n=3$ embryos per genotype). g-j pSmad immunohistochemistry on e10.5 coronal sections. Only simultaneous, but not individual loss of $L m \times 1 a$ and $1 b$ significantly reduced pSmad intensity in the DMe $\left(^{\star \star \star} p \leq 0.001\right.$ for $L m \times 1 a-/-; b-/-$ versus wild type, Lmx1a-/-;b-/- versus $L m \times 1 a-/-$, and $L m \times 1 a-/-; b-/-$ versus $L m \times 1 b-/-$ comparisons, oneway ANOVA with Tukey's post hoc test, $n=3$ wild-type embryos, $n=3$ Lmx1a-/- embryos, $n=3$ Lmx1b-/- embryos, and $n=4$ Lmx1a-/ $; b-/-$ embryos). All data are mean \pm s.d. Source data for panels $\mathbf{f}$, $\mathbf{k}$ are provided as Source Data File. Scale bars: $200 \mu \mathrm{m}(\mathbf{b}-\mathbf{e}) ; 100 \mu \mathrm{m}(\mathbf{g}-\mathbf{j})$

increase in pial surface as seen in naturally gyrencephalic mammals ${ }^{3}$. Additionally, there was a profound loss of cortical lamination in these transgenic mice ${ }^{31,49}$. Further, mice with permanently activated $\mathrm{Wnt} / \beta$-catenin signaling demonstrated a decrease in the number of BPs, rather than an increase as observed in higher mammals ${ }^{39}$.

Early constitutive ablation of $\beta$-catenin in the developing mouse telencephalon increased the numbers of both bRG and IPs but was insufficient to induce gyrification ${ }^{29,39}$. Although both bRG and IPs were increased in the number in the telencephalon of $\beta$-catenin ablated mice, IPs were expanded only at early stages likely because by the peak of neurogenesis, APs were severely depleted and could not further support the generation of excessive IPs ${ }^{29}$.

In contrast, in $\operatorname{Lm} \times 1 a-/-; b-/-$ mutants, reduction of Wnt/ $\beta$ catenin signaling occurred only after its transient overactivation and the resultant overexpansion of AP pool at earlier stages. When Wnt signaling was reduced at e15.5, at the peak of cortical neurogenesis, this expanded AP population was apparently sufficient to simultaneously produce enough bRG and IPs to drive cortical gyrification. Consistently with this hypothesis, suppression of the early activation of $\mathrm{Wnt} / \beta$-catenin signaling by electroporating Wnt inhibitor $D k k 1$ into the cerebral cortex, reduced the number of cortical APs, bRG and IPs and restored lissencephaly in $L m \times 1 a-/-; b-/-$ mice, revealing a critical role of Wnt signaling in the development of cortical gyrification in this mutant (Fig. 4).

Interestingly, despite local overexpansion of BPs in the emerging gyri in e15.5 Lmx1a-/-;b-/- embryos, we could not identify differences in progenitor behavior in different cortical regions in e12.5 Lmx1a-/-;b-/- mice. Thus, location of gyri and sulci in $L m x 1 a-/-; b-/-$ mice is likely determined by region-specific responses of initially expanded APs to reduced Wnt signaling at later stages, possibly resulting in differential delamination and/or migration of BPs. The molecular basis of differential response of different cortical regions to secreted factors remains unknown. Notably, however, we have recently reported that in another cortical gyrification mouse model, subtle changes in polarity at the apical ventricular surface coincide with location of gyri and are accompanied by localized accumulation of nuclear YAP, which is essential to drive localized proliferation of $\mathrm{BPs}^{21}$ 


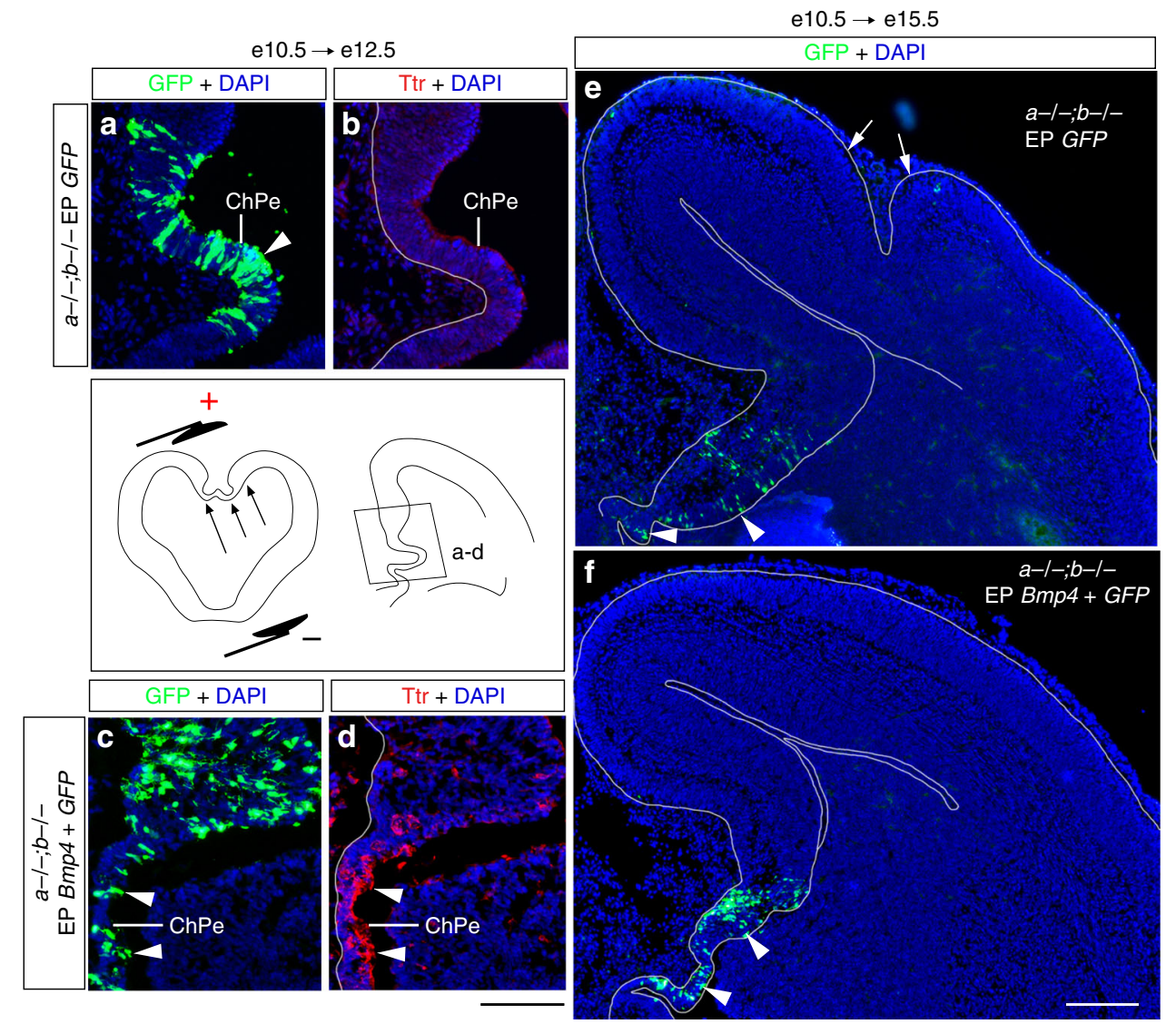

Fig. 6 Bmp4 rescues DMe development and cortical gyrification in $L m x 1 a-/-; b-/-$ mutants. Bmp4 and/or GFP expressing plasmids were electroporated into DMe of $L m \times 1 a-/-; b-/-$ embryos at e10.5 and analyzed at e12.5 (a-d) or e15.5 (e, f). To electroporate DMe, the positive electrode was placed against this region, as shown in the schematic below panel a. Negatively charged DNA moves toward the positive electrode (arrows) entering DMe. a-d Show DMe (a region boxed in the diagram below panel b). Immunohistochemistry revealed that Bmp4, but not GFP, induced Ttr in the Lmx1a-/-; $b-/-$ ChPe (arrowheads in d). GFP + ChPe cells (arrowheads in a, c) confirm successful electroporation of ChPe. e, f While GFP-electroporated Lmx1a-/-;b-/- mutants had cortical gyrification at e15.5 (e, arrows point to cortical gyri), electroporation of Bmp4 into DMe restored cortical lissencephaly (f). Arrowheads point to GFP+ cells in the DMe, showing specific electroporation of this domain. Scale bars: $100 \mu \mathrm{m}(\mathbf{a}-\mathbf{d}) ; 200 \mu \mathrm{m}(\mathbf{e}, \mathbf{f})$

In the canonical Wnt pathway, Wnt ligands bind to LRP5/ Frizzled receptors leading to translocation of $\beta$-catenin to the nucleus and activation of transcription of Wnt- $\beta$-catenin responsive genes ${ }^{50}$. In $L m x 1 a-/-; b-/-$ mutants, the rate of change of Wnt signaling, as revealed by expression levels of canonical Wnt pathway downstream genes Lef1 and Axin2, was fast: the switch from high to low Wnt signaling happened within 3 days (from e12.5 to e15.5), leading to dramatic changes in cortical progenitor populations. Fast effects of misregulated Wnt signaling have also been described in other experimental systems. For example, activating or reducing activity of Wnt pathway resulted in dramatic biological responses in wild-type chicken spinal cord or mouse neocortex already $24 \mathrm{~h}$ after electroporation of expression plasmids $\mathrm{s}^{39,51}$ highlighting that tight regulation of Wnt signaling is critical for normal development of the central nervous system. Analysis of Lef1 expression or an Axin2 reporter revealed a Wnt signaling gradient that in the wild-type telencephalon extends through neuroepithelium from the DMe (high) to lateral neocortex $(\text { low })^{40,52}$ (Supplementary Fig. 11a). The existence of a Wnt response gradient through the cortical neuroepithelium suggests that DMe-derived Wnt ligands diffuse (or are actively transported) via neuroepithelium. In e12.5 Lmxla-/-b-/mutants, a medio-lateral Lef1 expression gradient was still observed; however, it was brighter than in comparably staged wild-type controls (Supplementary Fig. 11a, b), as expected by the presence of an enlarged Wnt $3 a$-expressing DMe domain. Previously, several Wnts have been also detected in mouse and human cerebrospinal fluid (CSF) ${ }^{53,54}$. Thus, we cannot exclude a possibility that in Lmx1a-/-;b-/- mutants, some Wnt molecules reach neocortex via CSF as well.

Importantly, rescue of the DMe patterning in Lmxla-/-;b-/mutants by early electroporation of Bmp4 into the DMe was sufficient to normalize activity of the Wnt- $\beta$-catenin signaling in the distantly located neocortex to restore lissencephaly. These data confirm that the DMe was the primary source of a gyrificationrelated Wnt signaling in Lmxla-/-;b-/- mutants. Our experiments, however, do not exclude a possibility that other signaling molecules, beyond Wnt3a from the DMe, contribute to cortical gyrification in $L m \times 1 a / b$ double mutants. For example, signaling from lateral mesenchyme located adjacent to the neocortical primordium may directly contribute to cortical developmental abnormalities in $L m x 1 a / b$ mutants. However, we consider this an unlikely possibility since cortical gyrification was not detected in $L m \times 1 b-/-$ mutants. Thus, even if $L m \times 1 b$-dependent signaling from lateral mesenchyme contributes to cortical gyrification in Lmxla-/-;b-/- mutants, it still does so in cooperation with the DMe-derived Wnt signaling, which is dependent on both $L m x 1$ genes. Therefore, our experiments combining $L m \times 1 a / b$ mutants analysis with Bmp4/Dkk1 electroporation studies identify misregulation of DMe-derived Wnt signaling as a major contributor to the cortical phenotype of $L m \times 1 a-/-; b-/-$ mutants, 
a

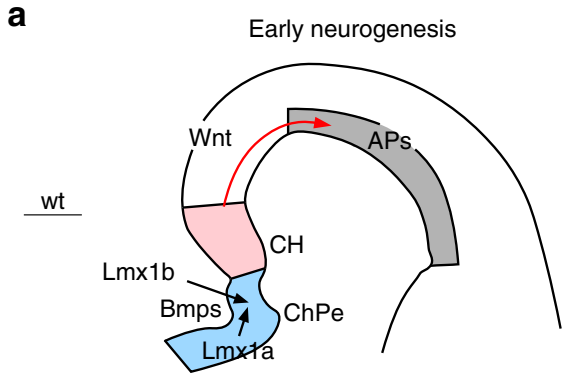

b

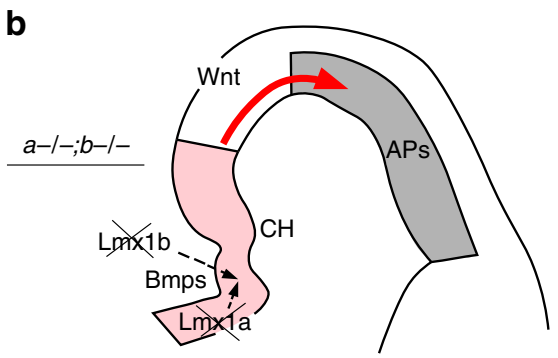

C

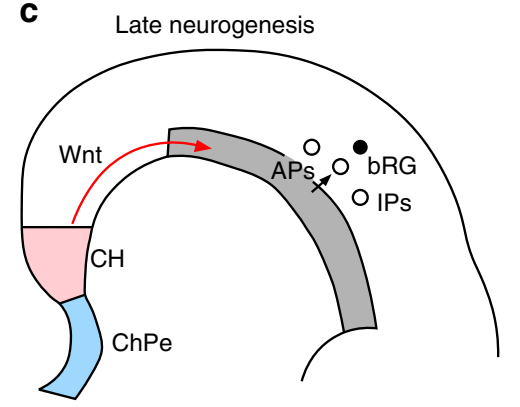

d

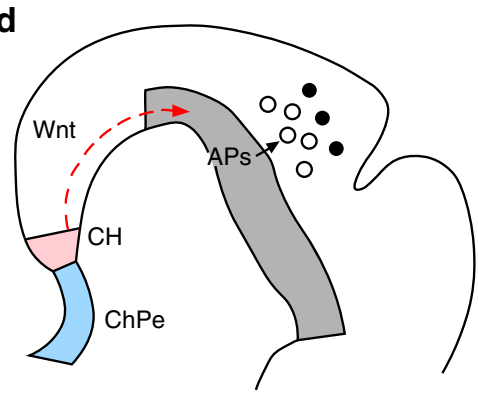

Fig. $7 \mathrm{Lm} \times 1 a / b$-dependent dorsomedial tissue interactions regulate cortical gyrification. a In early wild-type embryos, $L m \times 1 a$ and $L m \times 1 b$ are expressed in the telencephalic DMe and adjacent mesenchyme, respectively, and promote expression of secreted Bmps within their respective territories. Appropriate level of Bmp signaling specifies ChPe and $\mathrm{CH}$ in the DMe. Wnt3a signaling from the $\mathrm{CH}$ (red arrow) promotes proliferation of APs in the cortical VZ. Although we show Wnt signaling by arrow extending through neuroepithelium, our data cannot exclude a possibility that Wnt molecules are delivered to neocortical primordium via CSF. $\mathbf{b}$ Simultaneous loss of $L m x 1 a$ and $1 b$ reduces Bmp expression in both the mesenchyme and DMe, decreasing the level of Bmp signaling in the DMe. This results in DMe overproliferation and mispatterning, creating an enlarged Wnt3a expression domain at the DM in early Lmx1a-/-;b-/embryos. The excessive secreted Wnt3a (large red arrow) overactivates canonical Wnt- $\beta$-catenin signaling in distal cortical primordium, leading to excessive expansion of APs in Lmx1a-/-;b-/- mutants. c At later developmental stages, in wild-type embryos, Wnt- $\beta$-catenin signaling (red arrow) limits production of IPs and bRG from APs ${ }^{29}$. d By e15.5, normal DMe specification is restored in $L m x 1 a-/-; b-/-$ embryos, but enhanced apoptosis reduces the size of $\mathrm{CH}$ and DM-derived Wnt signaling (dashed red line). Reduced Wnt signaling allows excessive production of IPs and bRG from an earlier expanded AP population, resulting in cortical gyrification

identifying a mesenchymal/DMe Lmxla/1b-Bmp-Wnt pathway as a regulator of cortical gyrification.

Our study clearly demonstrates that lissencephaly in mice is maintained by redundant function of two $\operatorname{Lmx} 1$ genes. Genetic redundancy is believed to be maintained in evolution to stabilize key developmental processes in animal populations to make them less susceptible to various influences such as environmental conditions or genetic background ${ }^{55}$. Given the important role of DMe for cortical organization and the profound effect of relatively subtle variations of Bmp signaling on DMe development, it seems logical that the development of this particular tissue is stabilized via redundant pathways. Notably, the only two other genes known to maintain lissencephaly in the mouse (or any other lissencephalic species) are Flrt1 and Flrt3 which redundantly regulate neuronal adhesion during migration ${ }^{25}$. We conclude that lissencephaly is actively regulated during development and protected by redundant mechanisms. Disruption of these mechanisms is sufficient to induce gyrification of normally lissencephalic mouse neocortex, revealing striking plasticity of neocortical development in mice.

Similar to the developing neocortex of naturally gyrencephalic mammals, we observed local expansion of BPs in the emerging gyri but did not detect specific increases in apoptosis in cortical regions developing sulci in e15.5 $\operatorname{Lm} \times 1 a-/-; b-/-$ embryos. Taken together, these results suggest localized expansion of BPs as a major mechanism of cortical gyrification in $\operatorname{Lm} x 1 a-1-; b-1-$ mutants, although we cannot exclude a possibility that transient region-specific misregulation of apoptosis, for example at earlier stages, also contributes to the phenotype. In higher mammals, cortical gyrification is a complex process that beyond localized overexpansion of BPs, involves other mechanisms as well, such as region-specific differences in neuronal migration ${ }^{2-4,25}$. It remains to be investigated whether other cellular mechanisms that regulate cortical gyrification in higher mammals also contribute to cortical folding in Lmx1a-/-;b-/- mutants.

In conclusion, we have identified a redundant Lmxla/b-Bmpdependent pathway that shapes the dynamics of dorsal midline Wnt signaling. In mice, this pathway operates to prevent differential expansion of cortical progenitors and suppress gyrification of the distant neocortex. It has been recently demonstrated that similar to the mouse, a Wnt3a-expressing signaling center is also present in the DMe of the naturally gyrencephalic ferret ${ }^{56}$. Although the mechanisms that regulate DMe development and signaling in gyrencephalic mammals remain unknown, in the developing human neocortex, expression of Wnt receptor FZD8 is higher than in the mouse and even the chimpanzee, potentially making the developing human neocortex particularly sensitive to dynamics of Wnt signaling 57 . An important next step will be to determine whether modification of the DMe/mesenchymal signaling pathways that we describe here promote gyrification during evolution of naturally gyrencephalic mammals.

\section{Methods}

Mice. $L m x 1 a$-null $\left(L m x 1 a^{d r J}\right.$, The Jackson Laboratory strain \#000636) ${ }^{58}, L m x 1 b$ null $(L m \times 1 b-/-)^{59}, L m \times 1 b^{L a c Z 60}, L m \times 1 a-C r e^{61}$, and ROSA26-YFP 62 (The Jackson Laboratory strain \#006148) mice were used in this study. Mice were maintained on a mixed genetic background comprising C57Bl6, 129, FVB, and CD1. Noon of the day of the plug was considered e0.5. Embryos of both sexes were used for experiments. For cell proliferation experiments, pregnant females were intraperitoneally injected with BrdU at $50 \mathrm{mg} / \mathrm{kg}$. All animal experiments were performed in accordance with the protocols approved by the Institutional Animal Care and 
Use Committees of the University of Tennessee Health Science Center (UTHSC), Seattle Children's Research Institute or the University of Chicago.

Tissue analysis. Immunohistochemistry was performed using cryosections ${ }^{63}$. Embryos were collected in cold phosphate-buffered saline (PBS). Whole heads or dissected brains were fixed in freshly prepared $4 \%$ paraformaldehyde (PFA) in PBS for 1-12 h., washed three times in PBS (30 min each), equilibrated in 30\% sucrose in PBS for $2 \mathrm{~h}$ at $4{ }^{\circ} \mathrm{C}$, and embedded in optimum cutting temperature (OCT) compound (Tissue Tek). OCT blocks were sectioned on a cryostat at $12 \mu \mathrm{m}$. Sections were collected on Superfrost Plus glass slides (Fisher Scientific) and kept at $-20^{\circ} \mathrm{C}$. Then, sections were dried at room temperature for $20 \mathrm{~min}$, washed in PBS, blocked in PBS containing 1\% normal goat serum and $0.1 \%$ Triton X-100, and incubated with primary antibodies overnight at $4{ }^{\circ} \mathrm{C}$. Secondary antibodies were applied at room temperature for $1 \mathrm{~h}$. Some sections were counterstained with DAPI (Sigma-Aldrich) to visualize tissue. Sections were mounted in Fluorogel (EMS) and coverslipped. For BrdU and Ki67 immunostaining, slides were boiled in $1 \times$ target retrieval solution (Dako) for 10 min before the blocking step. For pSmad immunostaining, slides were incubated in the same solution for $7 \mathrm{~min}$ at $95{ }^{\circ} \mathrm{C}$. The following primary antibodies were used: anti-Lmxla (goat, Santa-Cruz, catalog \#sc54273, 1:500, RRID:AB 2136830), anti-Cux1 (rabbit, Santa-Cruz, catalog \#sc13024, 1:200, RRID:AB_2261231), anti-Ctip2 (rat, Abcam, catalog \#ab18465, 1:500, RRID:AB_2064130), anti-GFP antibody that recognizes YFP (chicken, catalog \#ab13970 Abcam, 1:500, RRID:AB_300798), anti-Reelin (mouse, Chemicon, cata$\log$ \#Mab5364, 1:500, RRID:AB_2179313), anti-BrdU (rat, Abcam, catalog \#ab6326, 1:50, RRID:AB_305426), anti-BrdU (mouse, Rockland, catalog \#600-401c29, 1:300, RRID:AB_10893609), anti-Ki67 (mouse, BD Pharmingem, catalog \#556003, 1:250, RRID:AB_396287), anti-Pax6 (rabbit, Covance, catalog \#Prb-278p100, 1:300, RRID:AB 291612), anti-Tbr2 (rat, eBioscience, catalog \#14-487582, 1:200, RRID:AB_11042577), anti- $\beta$-catenin (mouse, BD Biosciences, catalog \#610153, 1:200, RRID:AB_397554), anti-Lef1 (rabbit, Cell Signaling Technology, catalog \#2230S, 1:300), anti-pSmad (rabbit, Chemicon, catalog \#AB3848, 1:50, RRID:AB_177439), anti-Ttr (rabbit, Dako, catalog \#A0002, 1:200, RRID: AB_2335696), anti-activated Caspase 3 (rabbit, Promega, catalog \#G7481, 1:250, RRID:AB_430875), and anti-p73 (mouse, Fisher, catalog \#MA5-14117, 1:100, RRID:AB_10987160) with species-specific secondary antibodies conjugated with Alexa 350, 488, 568, or 594 fluorophores (Life Technologies).

For Nissl staining, sections were incubated in $0.1 \%$ cresyl violet solution for $10 \mathrm{~min}$, dehydrated through an ethanol/xylens series and coverslipped with Permount (Fisher) ${ }^{64}$. In situ hybridization was performed using digoxigeninlabeled $L m \times 1 a^{26}, L m x 1 b^{26}, B m p 4^{65}, T t r^{66}$, and $W n t 3 a^{32}$ RNA probes and antidigoxigenin antibody conjugated with alkaline phosphatase (Roche).

Hybridizations were performed in a buffer containing $50 \%$ formamide, $4 \times$ saline sodium citrate, and $1 \%$ sodium dodecyl sulfate at $70{ }^{\circ} \mathrm{C}$ overnight. Color reactions were carried out using NBT/BCIP substrates (4-nitroblue tetrazolium chloride/5bromo-4-chloro-3-idolyl phosphate ${ }^{67}$. X-gal staining was performed using sections from fresh-frozen embryos ${ }^{68}$. Slides were fixed in $0.2 \%$ glutaraldehyde in PBS for $10 \mathrm{~min}$ on ice and after brief rinsing were incubated in X-gal staining solution $(0.02 \%$ Igepal, $0.01 \%$ sodium deoxycholate, $5 \mathrm{mM}$ potassium ferricyanide, $5 \mathrm{mM}$ potassium ferrocyanide, and $2 \mathrm{mM} \mathrm{MgCl}$ diluted in $0.1 \mathrm{M}$ phosphate buffer) at $37^{\circ} \mathrm{C}$ overnight in the dark. To visualize tissue, X-gal-stained sections were counterstained with Nuclear Fast Red (Sigma).

Images of immunostained sections (except $\beta$-catenin-stained sections) were taken using a Zeiss AxioImager A2 microscope equipped with an AxioCam Mrm camera and AxioVision Rel 4.9 software (Zeiss). Images of $\beta$-cateninimmunostained sections were taken using a Zeiss LCM 710 laser scanning confocal microscope with Zen software. Brightfield images were taken using a Leica MZFLIII microscope with Leica DFC425 camera and LAS V3.8 software or Olympus SZX microscope with Leica MC170HD camera and LAS V4.5 software. Figure panels were assembled in Photoshop CC 2018 (Adobe).

\section{Mouse in utero electroporation. Plasmids encoding GFP, Dkk1, and Bmp4} $(1 \mu \mathrm{g} / \mu \mathrm{l})$ were injected using a fine-glass microcapillary into a lateral ventricle and electroporated into either telencephalic DMe or the neocortical primordium using paddle electrodes with BTX ECM830 electroporator ${ }^{69}$. To target the telencephalic DMe, the positive electrode was placed adjacent to the DMe, as shown in Fig. 6 (in schematic below Fig. 6a). The neocortical primordium was targeted by placing the positive electrode against the neocortical wall. To facilitate injections, Fast Green dye was added to plasmid solutions. The following plasmids were used: $p C I G-$ mouse $D k k 1^{39}, p C A G G S$-mouse $B m p 4^{70}$, and $p C A G G S$ - $G F P^{71}$. After electroporation, the uterus was returned to abdomen, and the embryos continued development. Upon collection, embryos were evaluated under a fluorescence stereo microscope, and those showing GFP fluorescence in appropriate brain regions were serially sectioned on a cryostat and used for analysis.

Laser capture microdissection (LCM) and qRT-PCR. Embryonic heads were flash-frozen in OCT on dry ice and coronally sectioned on a cryostat at $10 \mu \mathrm{m}$. Sections for LCM were mounted on uncharged glass slides (AmLabs ${ }^{\mathrm{TM}}$ ), dried at room temperature for $30 \mathrm{~s}$, and stained with hematoxylin and eosin to visualize tissue morphology, using HistoGene LCM Frozen Sections Staining kit (Fisher
Scientific, Cat \#KIT0401) ${ }^{72}$. LCM was performed using an ArcturusXT laser capture microdissection machine with Arcturus CapSure Macro LCM caps. For Bmp expression analysis, five telencephalic sections separated from each other by $10 \mu \mathrm{m}$ were used for LCM from each e10.5 embryo. Adjacent sections were immunostained against Lmxla and used as templates to localize boundaries of the telencephalic DMe. For analysis of mesenchyme, mesenchymal tissue located above the DMe was microdissected (green domain in Fig. 5a). For Axin2 expression analysis in e12.5 and e15.5 embryos, cortical primordium located between the dorsal cortical bend and LGE (gray region in schematics adjacent to Supplementary Fig. 11c and Supplementary Fig. 12b) was microdissected from 4 to 6 non-adjacent telencephalic sections from each embryo. For gene expression analysis in Bmp rescue experiments (Supplementary Fig. 16), sections of pCAGGS-Bmp4 and/or $p C A G G S$ $G F P$-electroporated embryos were first evaluated under a fluorescence microscope and embryos that specifically expressed GFP in the DMe (similar to those shown in Fig. 6e, f) were identified. ChPe and neocortical primordium from sections from these embryos were isolated by LCM and used for gene expression analysis.

RNA from microdissected samples was isolated using Pico Pure RNA purification kit (Arcturus KIT\#0204). Before eluting RNA, RNAse free DNAse I (Qiagen) was applied to each column and incubated for $15 \mathrm{~min}$ at room temperature to remove traces of genomic DNA. cDNA was generated using cDNA synthesis kit (Biorad, cat\#1708890). qRT-PCR was performed on Roche LC480 Real-time PCR machine with SYBR Fast qPCR master mix (Kapa Biosystems) $)^{72,73}$. Transcript levels were normalized to Gapdh. All samples were tested in triplicates. At least three embryos per genotype per experimental condition were tested using primers listed in Supplementary Table 1.

Cell counts, measurements, and statistical analysis. For each embryo, the telencephalon was coronally serially sectioned and the same number of sections was placed on each slide to identify the position of each section along the anterior-posterior (A-P) axis of the telencephalon. For consistency, all comparisons between mutant and control embryos were performed using sections taken at similar A-P levels, approximately midway along the A-P axis of the telencephalon. At least three embryos per genotype or experimental condition (typically three non-adjacent sections per embryo) were evaluated in each analysis at each developmental stage.

Cortical parameters (area, regional thickness of cortical neuroepithelium) were measured between the telencephalic dorsal bend (pointed by upper arrow in Supplementary Fig. 1e) and LGE (pallial-subpallial boundary) (lower arrow in Supplementary Fig. 1e $)^{74-76}$, using ImageJ (NIH). For each embryo, cortical area in left and right hemispheres was added to generate a total cortical area, which was compared between $L m \times 1 a-/-; b-/-$ and control littermates (Supplementary Fig. 1f). For clarity, cortical area in $L m x 1 a-/-; b-/-$ mutants was normalized to that in wild-type controls. To evaluate whether cortical gyri occupy non-random positions or are randomly distributed throughout e18.5 Lmx1a-/-;b-/neocortex, the cortical ventricular surface (between the dorsal cortical bend and LGE) was divided into 10 equally sized segments (Supplementary Fig. 1e). Cortical thickness was measured in the middle of each segment in different embryos and plotted (Supplementary Fig. 1g, h). Cortical progenitors were counted in $100-\mu \mathrm{m}-$ wide radial segments (columns) spanning the entire thickness of the neocortex, from ventricular to outer cortical surface. For consistency, in $L m \times 1 a-/-; b-/-$ embryos, cell counts were performed in dorsal neocortical primordium, which develops a dorsal bulge, the most consistent cortical gyrus in $\operatorname{Lm} \times 1 a-/-; b-/-$ embryos (Supplementary Fig. lb-d, h). In control embryos, cell counts were performed in the same region of the cortex as in $\operatorname{Lm} x 1 a-/-; b-/-$ mutants. IPs were defined as Tbr2 + cells $^{16,29}$. Originally, bRG cells were defined as Pax6+/Tbr2 - progenitors that occupy the SVZ and possess a single basal process $^{8,9}$. More recently, however, bRG cells with diverse morphology were described, indicating that apical-only process is not a definitive feature of $\mathrm{bRG}^{11,77}$ Thus, similar to Wang et al. ${ }^{16}$ and Draganova et al. ${ }^{29}$, we identified bRG as Pax6+l Tbr2 - cells located basal to the Tbr2+IP band. Similar to wild-type controls, in Lmx1a-/-;b-/- mutants, these basal Pax6+/Tbr2 - cells were Ki67+ and incorporated BrdU after a short BrdU pulse (Supplementary Fig. 3a-e, g-i), indicating that they indeed were proliferating progenitors. To compare distribution of proliferating cells in e15.5 wild type and Lmx1a-/-;b-/- embryos (Supplementary Fig. 3k), a 90 min BrdU pulse was performed and fractions of basally located proliferating (S-phase) cells (the number of BrdU+ cells located beyond the Pax6+VZ divided by the total number of BrdU+ cells) were calculated. To evaluate activity of the Bmp signaling pathway in the telencephalic DMe of e10.5 embryos, intensity of pSmad immunostaining was measured using ImageJ $(\mathrm{NIH})^{33}$. The boundaries of the DMe were identified using adjacent Lmxlaimmunostained sections as templates. To analyze cell-cycle exit, embryos were harvested $20-24 \mathrm{~h}$ after a BrdU pulse and the fraction of cells that exited the cell cycle (the number of BrdU+/Ki67 - cells divided by the total number of BrdU+ cells) was calculated in the neocortex ${ }^{78}$

Statistical analysis was performed using IBM SPSS Statistics (version 25) software. All quantitative data are presented as the mean \pm s.d. Statistical analysis between two groups was performed using two-tailed $t$-test. Multiple groups were analyzed using one-way ANOVA with Tukey's post hoc test. Bonferroni correction was applied when multiple comparisons were performed. $P<0.05$ was considered statistically significant. 
Reporting summary. Further information on research design is available in the Nature Research Reporting Summary linked to this article.

\section{Data availability}

The data that support the findings of this study are available from the corresponding authors upon reasonable request. Source data for Fig. lk, n; 2f, i, l, o, r, u; 4f-h; 5f, k and Supplementary Figs. 1f-h; 3k-o, r; 4d; 7e; 9g, l; 11c, d; 12b, c; 15 and 16b, c are provided as a Source data file.

Received: 25 February 2019; Accepted: 4 October 2019; Published online: 15 November 2019

\section{References}

1. Rakic, P. Evolution of the neocortex: a perspective from developmental biology. Nat. Rev. Neurosci. 10, 724-735 (2009).

2. Geschwind, D. H. \& Rakic, P. Cortical evolution: judge the brain by its cover. Neuron 80, 633-647 (2013).

3. Borrell, V. How cells fold the cerebral cortex. J. Neurosci. 38, 776-783 (2018).

4. Llinares-Benadero, C. \& Borrell, V. Deconstructing cortical folding: genetic, cellular and mechanical determinants. Nat. Rev. Neurosci. 20, 161-176 (2019).

5. Lewitus, E., Kelava, I., Kalinka, A. T., Tomancak, P. \& Huttner, W. B. An adaptive threshold in mammalian neocortical evolution. PLoS Biol. 12, e1002000 (2014).

6. O'Leary, M. A. et al. The placental mammal ancestor and the post-K-Pg radiation of placentals. Science 339, 662-667 (2013).

7. Kelava, I. et al. Abundant occurrence of basal radial glia in the subventricular zone of embryonic neocortex of a lissencephalic primate, the common marmoset Callithrix jacchus. Cereb. Cortex 22, 469-481 (2012).

8. Hansen, D. V., Lui, J. H., Parker, P. R. \& Kriegstein, A. R. Neurogenic radial glia in the outer subventricular zone of human neocortex. Nature 464, 554-561 (2010).

9. Lui, J. H., Hansen, D. V. \& Kriegstein, A. R. Development and evolution of the human neocortex. Cell 146, 18-36 (2011).

10. Borrell, V. \& Götz, M. Role of radial glial cells in cerebral cortex folding. Curr. Opin. Neurobiol. 27, 39-46 (2014)

11. Taverna, E., Götz, M. \& Huttner, W. B. The cell biology of neurogenesis: toward an understanding of the development and evolution of the neocortex. Annu. Rev. Cell Dev. Biol. 30, 465-502 (2014).

12. Fernández, V., Llinares-Benadero, C. \& Borrell, V. Cerebral cortex expansion and folding: what have we learned? EMBO J. 35, 1021-1044 (2016).

13. Stahl, R. et al. Trnp1 regulates expansion and folding of the mammalian cerebral cortex by control of radial glial fate. Cell 153, 535-549 (2013).

14. Florio, M. et al. Human-specific gene ARHGAP11B promotes basal progenitor amplification and neocortex expansion. Science 347, 1465-1470 (2015).

15. Ju, X. C. et al. The hominoid-specific gene TBC1D3 promotes generation of basal neural progenitors and induces cortical folding in mice. Elife 5, e18197 (2016).

16. Wang, L., Hou, S. \& Han, Y. G. Hedgehog signaling promotes basal progenitor expansion and the growth and folding of the neocortex. Nat. Neurosci. 19, 888-896 (2016).

17. Liu, J. et al. The primate-specific gene TMEM14B marks outer radial glia cells and promotes cortical expansion and folding. Cell Stem Cell 21, 635-649 (2017).

18. Fiddes, I. T. et al. Human-specific NOTCH2NL genes affect Notch signaling and cortical neurogenesis. Cell 173, 1356-1369 (2018).

19. Suzuki, I. K. et al. Human-specific NOTCH2NL genes expand cortical neurogenesis through Delta/Notch regulation. Cell 173, 1370-1384 (2018).

20. Smith, R. S. et al. Sodium channel SCN3A $\left(\mathrm{Na}_{\mathrm{V}} 1.3\right)$ regulation of human cerebral cortical folding and oral motor development. Neuron 99, 905-913 (2018).

21. Roy, A. et al. PI3K-Yap activity drives cortical gyrification and hydrocephalus in mice. Elife 8, e45961 (2019).

22. Matsumoto, N., Shinmyo, Y., Ichikawa, Y. \& Kawasaki, H. Gyrification of the cerebral cortex requires FGF signaling in the mammalian brain. Elife 6, e29285 (2017).

23. Rash, B. G., Tomasi, S., Lim, H. D., Suh, C. Y. \& Vaccarino, F. M. Cortical gyrification induced by fibroblast growth factor 2 in the mouse brain. $J$. Neurosci. 33, 10802-10814 (2013).

24. Rash, B. G., Lim, H. D., Breunig, J. J. \& Vaccarino, F. M. FGF signaling expands embryonic cortical surface area by regulating Notch-dependent neurogenesis. J. Neurosci. 31, 15604-15617 (2011).

25. Del Toro, D. et al. Regulation of cerebral cortex folding by controlling neuronal migration via FLRT adhesion molecules. Cell 169, 621-635 (2017).
26. Mishima, Y., Lindgren, A. G., Chizhikov, V. V., Johnson, R. L. \& Millen, K. J. Overlapping function of Lmxla and Lmxlb in anterior hindbrain roof plate formation and cerebellar growth. J. Neurosci. 29, 11377-11384 (2009).

27. Doucet-Beaupré, H., Ang, S. L. \& Lévesque, M. Cell fate determination, neuronal maintenance and disease state: the emerging role of transcription factors Lmxla and Lmx1b. FEBS Lett. 89, 3727-3738 (2015).

28. Itoh, Y. et al. PDK1-Akt pathway regulates radial neuronal migration and microtubules in the developing mouse neocortex. Proc. Natl. Acad. Sci. USA 113, E2955-E2964 (2016).

29. Draganova, $K$. et al. Wnt $/ \beta$-catenin signaling regulates sequential fate decisions of murine cortical precursor cells. Stem Cells 33, 170-182 (2015).

30. Nowakowski, T. J. et al. MicroRNA-92b regulates the development of intermediate cortical progenitors in embryonic mouse brain. Proc. Natl Acad. Sci. USA 110, 7056-7061 (2013).

31. Chenn, A. \& Walsh, C. A. Regulation of cerebral cortical size by control of cell cycle exit in neural precursors. Science 297, 365-369 (2002).

32. Grove, E. A., Tole, S., Limon, J., Yip, L. \& Ragsdale, C. W. The hem of the embryonic cerebral cortex is defined by the expression of multiple Wnt genes and is compromised in Gli3-deficient mice. Development 125, 2315-2325 (1998).

33. Cheng, X. et al. Central roles of the roof plate in telencephalic development and holoprosencephaly. J. Neurosci. 26, 7640-7649 (2006).

34. Fernandes, M., Gutin, G., Alcorn, H., McConnell, S. K. \& Hébert, J. M. Mutations in the BMP pathway in mice support the existence of two molecular classes of holoprosencephaly. Development 134, 3789-3794 (2007)

35. Voss, A. K., Krebs, D. L. \& Thomas, T. C3G regulates the size of the cerebral cortex neural precursor population. EMBO J. 25, 3652-3663 (2006).

36. Ivaniutsin, U., Chen, Y., Mason, J. O., Price, D. J. \& Pratt, T. Adenomatous polyposis coli is required for early events in the normal growth and differentiation of the developing cerebral cortex. Neural Dev. 4, 3 (2009).

37. Barber, M. \& Pierani, A. Tangential migration of glutamatergic neurons and cortical patterning during development: lessons from Cajal-Retzius cells. Dev. Neurobiol. 76, 847-881 (2016).

38. Imayoshi, I., Shimogori, T., Ohtsuka, T. \& Kageyama, R. Hes genes and neurogenin regulate non-neural versus neural fate specification in the dorsal telencephalic midline. Development 135, 2531-2541 (2008).

39. Munji, R. N., Choe, Y., Li, G., Siegenthaler, J. A. \& Pleasure, S. J. Wnt signaling regulates neuronal differentiation of cortical intermediate progenitors. J. Neurosci. 31, 1676-1687 (2011).

40. Caronia-Brown, G., Yoshida, M., Gulden, F., Assimacopoulos, S. \& Grove, E. A. The cortical hem regulates the size and patterning of neocortex. Development 141, 2855-2865 (2014).

41. Mangale, V. S. et al. Lhx2 selector activity specifies cortical identity and suppresses hippocampal organizer fate. Science 319, 304-309 (2008).

42. Bielen, H. \& Houart, C. The Wnt cries many: Wnt regulation of neurogenesis through tissue patterning, proliferation, and asymmetric cell division. Dev. Neurobiol. 74, 772-780 (2014).

43. Borrell, V. \& Marín, O. Meninges control tangential migration of hem-derived Cajal-Retzius cells via CXCL12/CXCR4 signaling. Nat. Neurosci. 9, 1284-1293 (2006).

44. Siegenthaler, J. A. et al. Retinoic acid from the meninges regulates cortical neuron generation. Cell 139, 597-609 (2009).

45. Siegenthaler, J. A. \& Pleasure, S. J. We have got you 'covered': how the meninges control brain development. Curr. Opin. Genet. Dev. 21, 249-255 (2011).

46. Choe, Y., Siegenthaler, J. A. \& Pleasure, S. J. A cascade of morphogenic signaling initiated by the meninges controls corpus callosum formation. Neuron 73, 698-712 (2012).

47. Watanabe, M. et al. BMP4 sufficiency to induce choroid plexus epithelial fate from embryonic stem cell-derived neuroepithelial progenitors. J. Neurosci. 32, 15934-15945 (2012).

48. Camargo Ortega, G. et al. The centrosome protein AKNA regulates neurogenesis via microtubule organization. Nature 567, 113-117 (2019).

49. Chenn, A. \& Walsh, C. A. Increased neuronal production, enlarged forebrains and cytoarchitectural distortions in beta-catenin overexpressing transgenic mice. Cereb. Cortex 13, 599-606 (2003).

50. Logan, C. Y. \& Nusse, R. The Wnt signaling pathway in development and disease. Annu. Rev. Cell Dev. Biol. 20, 781-810 (2004).

51. Megason, S. G. \& McMahon, A. P. A mitogen gradient of dorsal midline Wnts organizes growth in the CNS. Development 129, 2087-2098 (2002).

52. Mutch, C. A., Schulte, J. D., Olson, E. \& Chenn, A. Beta-catenin signaling negatively regulates intermediate progenitor population numbers in the developing cortex. PLoS ONE 5, e12376 (2010).

53. Johansson, P. A. et al. The transcription factor Otx2 regulates choroid plexus development and function. Development 140, 1055-1066 (2013).

54. Kaiser, K. et al. WNT5A is transported via lipoprotein particles in the cerebrospinal fluid to regulate hindbrain morphogenesis. Nat. Commun. 10, 1498 (2019) 
55. Lagha, M., Bothma, J. P. \& Levine, M. Mechanisms of transcriptional precision in animal development. Trends Genet. 28, 409-416 (2012).

56. Jones, W. D., Guadiana, S. M. \& Grove, E. A. A model of neocortical area patterning in the lissencephalic mouse may hold for larger gyrencephalic brains. J. Comp. Neurol. 527, 1461-1477 (2019).

57. Boyd, J. L. et al. Human-chimpanzee differences in a FZD8 enhancer alter cellcycle dynamics in the developing neocortex. Curr. Biol. 25, 772-779 (2015).

58. Chizhikov, V. et al. Molecular definition of an allelic series of mutations disrupting the mouse Lmxla (dreher) gene. Mamm. Genome 17, 1025-1032 (2006).

59. Chen, H. et al. Limb and kidney defects in Lmxlb mutant mice suggest an involvement of LMX1B in human nail patella syndrome. Nat. Genet. 19, 51-55 (1998)

60. Schweizer, H., Johnson, R. L. \& Brand-Saberi, B. Characterization of migration behavior of myogenic precursor cells in the limb bud with respect to Lmxib expression. Anat. Embryol. 208, 7-18 (2004).

61. Chizhikov, V. V. et al. Lmxla regulates fates and location of cells originating from the cerebellar rhombic lip and telencephalic cortical hem. Proc. Natl Acad. Sci. USA 107, 10725-10730 (2010).

62. Srinivas, S. et al. Cre reporter strains produced by targeted insertion of EYFP and ECFP into the ROSA26 locus. BMC Dev. Biol. 1, 4 (2001)

63. Iskusnykh, I. Y., Steshina, E. Y. \& Chizhikov, V. V. Loss of Ptfla leads to a widespread cell-fate misspecification in the brainstem, affecting the development of somatosensory and viscerosensory nuclei. J. Neurosci. 36, 2691-2710 (2016).

64. Roy, A. et al. Mouse models of human PIK3CA-related brain overgrowth have acutely treatable epilepsy. Elife 4, e12703 (2015).

65. Jones, C. M., Lyons, K. M. \& Hogan, B. L. Involvement of Bone Morphogenetic Protein-4 (BMP-4) and Vgr-1 in morphogenesis and neurogenesis in the mouse. Development 111, 531-542 (1991).

66. Duan, W., Cole, T. \& Schreiber, G. Cloning and nucleotide sequencing of transthyretin (prealbumin) cDNA from rat choroid plexus and liver. Nucleic Acids Res. 17, 3979 (1989).

67. Bulchand, S., Subramanian, L. \& Tole, S. Dynamic spatiotemporal expression of LIM genes and cofactors in the embryonic and postnatal cerebral cortex. Dev. Dyn. 226, 460-469 (2003).

68. Kridsada, K. et al. Roof plate-derived radial glial-like cells support developmental growth of rapidly adapting mechanoreceptor ascending axons. Cell Rep. 23, 2928-2941 (2018).

69. Matsui, A., Yoshida, A. C., Kubota, M., Ogawa, M. \& Shimogori, T. Mouse in utero electroporation: controlled spatiotemporal gene transfection. J. Vis. Exp. 54, 3024 (2011).

70. Ohta, S., Wang, B., Mansour, S. L. \& Schoenwolf, G. C. BMP regulates regional gene expression in the dorsal otocyst through canonical and non-canonical intracellular pathways. Development 143, 2228-2237 (2016).

71. Ogawa, H., Inouye, S., Tsuji, F. I., Yasuda, K. \& Umesono, K. Localization, trafficking, and temperature-dependence of the Aequorea green fluorescent protein in cultured vertebrate cells. Proc. Natl Acad. Sci. USA 92, 11899-11903 (1995).

72. Iskusnykh, I. Y., Buddington, R. K. \& Chizhikov, V. V. Preterm birth disrupts cerebellar development by affecting granule cell proliferation program and Bergmann glia. Exp. Neurol. 306, 209-221 (2018).

73. Yoo, J. Y., Mak, G. K. \& Goldowitz, D. The effect of hemorrhage on the development of the postnatal mouse cerebellum. Exp. Neurol. 252, 85-94 (2014).

74. Pulvers, J. N. \& Huttner, W. B. Brcal is required for embryonic development of the mouse cerebral cortex to normal size by preventing apoptosis of early neural progenitors. Development 136, 1859-1868 (2009).

75. Fei, J. F., Haffner, C. \& Huttner, W. B. 3' UTR-dependent, miR-92-mediated restriction of Tis 21 expression maintains asymmetric neural stem cell division to ensure proper neocortex size. Cell Rep. 7, 398-411 (2014).
76. Chen, Q. et al. EphA4 regulates the balance between self-renewal and differentiation of radial glial cells and intermediate neuronal precursors in cooperation with FGF signaling. PLoS ONE 10, e0126942 (2015).

77. Namba, T. \& Huttner, W. B. Neural progenitor cells and their role in the development and evolutionary expansion of the neocortex. WIREs Dev. Biol. 6, e256 (2017).

78. Lavado, A. et al. Tumor suppressor Nf2 limits expansion of the neural progenitor pool by inhibiting Yap/Taz transcriptional coactivators. Development 140, 3323-3334 (2013).

\section{Acknowledgments}

We thank R. Johnson (MD Anderson Cancer Center), S. Pleasure (UCSF), S. Ohta and G. Schoenwolf (University of Utah), and B. Nelson (Seattle Children's) for mice, expression vectors, and plasmids for in situ probes, Y. Mishima (University of Chicago), C. Wang, J. Skibo (Seattle Children's), S. Krat, L. Mukhametzyanova, and A. Zakharova (UTHSC) for mouse genotyping. This work was supported by NIH grants T32GM07179 supporting A.G.L., R01 NS093009 and R21 NS077163 to V.V.C., R01 NS072441 and R01 NS099027 to K.J.M., and by the Neuroscience Institute, and Center of Integrative and Translational Genomics of the UTHSC.

\section{Author contributions}

V.V.C. and K.J.M. designed the study; V.V.C., I.Y.I., E.Y.S., N.F. A.G.L., A.S.S., and A.R. performed experiments and analyzed data; V.V.C., S.T., and K.J.M. supervised the study; V.V.C., A.S.S., A.R., S.T., and K.J.M. wrote the paper.

\section{Competing interests}

The authors declare no competing interests.

\section{Additional information}

Supplementary Information accompanies this paper at https://doi.org/10.1038/s41467 019-12913-z.

Correspondence and requests for materials should be addressed to V.V.C. or K.J.M.

Peer review information Nature Communications thanks Victor Borrell, Thomas Pratt and Alessandro Sessa for their contribution to the peer review of this work.

Reprints and permission information is available at http://www.nature.com/reprints

Publisher's note Springer Nature remains neutral with regard to jurisdictional claims in published maps and institutional affiliations.

Open Access This article is licensed under a Creative Commons Attribution 4.0 International License, which permits use, sharing, adaptation, distribution and reproduction in any medium or format, as long as you give appropriate credit to the original author(s) and the source, provide a link to the Creative Commons license, and indicate if changes were made. The images or other third party material in this article are included in the article's Creative Commons license, unless indicated otherwise in a credit line to the material. If material is not included in the article's Creative Commons license and your intended use is not permitted by statutory regulation or exceeds the permitted use, you will need to obtain permission directly from the copyright holder. To view a copy of this license, visit http://creativecommons.org/ licenses/by/4.0/

(C) The Author(s) 2019 